\title{
Age-Related Changes in Right Middle Frontal Gyrus Volume Correlate with Altered Episodic Retrieval Activity
}

\author{
M. Natasha Rajah, ${ }^{1}$ Rafael Languay, ${ }^{1}$ and Cheryl L. Grady ${ }^{2}$ \\ ${ }^{1}$ Douglas Mental Health University Institute and Department of Psychiatry, McGill University, Montreal, Quebec H4H 1R3, Canada, and ${ }^{2}$ Rotman Research \\ Institute, Baycrest Centre, University of Toronto, Toronto, Ontario M6A 2E1, Canada
}

\begin{abstract}
Age-related deficits in episodic retrieval have been associated with volume reductions in the middle frontal gyrus (MFG). However, it remains unclear how this age-related reduction in MFG volume correlates with neural activity during retrieval. To address this, we conducted in vivo volumetry of the frontal cortex in young and older human adults and found more volume loss on the right than on the left MFG with age. We then examined how left and right MFG volume correlated with fMRI activity during successful retrieval of item, spatial context, and temporal context information in both age groups. In young adults, larger right MFG volume was positively correlated with greater activity in a commonly found episodic retrieval network that included bilateral dorsolateral prefrontal cortex (DLPFC) and bilateral inferior parietal cortex. Within this network, left DLPFC and right inferior parietal cortex activity predicted memory performance. In older adults, a positive structure-function association in DLPFC for either left or right MFG/DLPFC was not observed. Instead, right MFG volume was negatively correlated with activity in several regions in older adults, including the parahippocampal cortex (PHC) and anterior cingulate. Less activity in the $\mathrm{PHC}$ region predicted better item memory, and less activity in the anterior cingulate predicted better spatial context accuracy in older adults. We conclude that age-related change in the structure-function association in MFG/DLPFC impacts retrieval activity and performance, and those older adults with larger right MFG volume attempt to compensate for this change by modifying activity in other brain regions to help retrieval performance.
\end{abstract}

\section{Introduction}

Healthy aging is associated with volume reductions and functional changes in the middle frontal gyrus (MFG) (Buckner et al., 2000; Raz et al., 2005). These changes in the MFG have been associated with age-related declines in episodic memory retrieval (Buckner et al., 2000; West, 2000; Cabeza, 2002): the ability to remember previously experienced items/events in rich contextual detail (Tulving, 1984). In particular, older adults exhibit deficits in retrieving contextual details from episodic memory. In young adults, context retrieval has been associated with bilateral MFG activity, and there is debate as to which cognitive processes left versus right MFG mediates during context retrieval (Dobbins et al., 2003; Knoch et al., 2006; Slotnick and Moo, 2006; Hayama and Rugg, 2009; Kaller et al., 2011). Our recent studies suggest that left and right MFG may mediate response selection and monitoring processes, respectively, during context retrieval (Rajah et al., 2008, 2010).

In a review of functional neuroimaging studies of healthy aging, we observed there was consistent under-recruitment of right

\footnotetext{
Received April 4, 2011; revised July 29, 2011; accepted Sept. 4, 2011

Author contributions: M.N.R. designed research; M.N.R. and R.L. performed research; M.N.R., R.L., and C.L.G. analyzed data; M.N.R. wrote the paper.

This study was funded by Canadian Institutes of Health Research (CIHR) Operating Grant MOP82036 awarded to M.N.R., a CIHR New Investigator award, and the Institute of Aging. We thank Y. Czechowska for her involvement in the volumetric analysis and D. Maillet for his assistance with the figures.

Correspondence should be addressed to Dr. M. Natasha Rajah, Douglas Hospital Research Centre, McGill University, 2147 Moe Levin Centre, Memory Clinic, 6875 LaSalle Boulevard, Verdun, QC H4H 1R3, Canada. E-mail: maria.rajah@mcgill.ca.

DOI:10.1523/JNEUROSCI.1690-11.2011

Copyright $\odot 2011$ the authors $\quad 0270-6474 / 11 / 3117941-14 \$ 15.00 / 0$
}

MFG during episodic retrieval with age (Rajah and D'Esposito, 2005). Moreover, age-related deficits in spatial and temporal context retrieval have been particularly linked with the functional under-recruitment of right MFG (Cabeza et al., 2000; Duarte et al., 2008; Rajah et al., 2010). This suggests that there may be a deficit in the right MFG with age, which impacts the recruitment of neural networks important for episodic retrieval, particularly context retrieval.

Evidence that healthy aging is associated with greater volume loss in right versus left MFG would lend support to this rightMFG-deficit hypothesis. However, previous in vivo volumetry studies have reported a linear decrease in bilateral lateral prefrontal cortex (PFC) gray matter (GM) volume with age (including MFG) (Jernigan et al., 2001; Tisserand et al., 2002; Raz et al., 2005). Raz et al. (2004) tested whether there was hemispheric asymmetry in lateral PFC volume loss with age and did not observe a significant effect. This may be because the lateral PFC region of interest extended across multiple frontal gyri and was not specific to the MFG (Raz et al., 2004).

In the current study, we test the hypothesis that healthy aging is associated with greater gray matter volume reductions in right versus left MFG, and that this in turn impacts retrieval-related activity in the MFG and other retrieval-related brain regions, and negatively influences context retrieval in older adults. We conducted volumetric segmentation of left and right MFG in healthy young and older adults who also performed an fMRI study of item, spatial context, and temporal context retrieval. We used multivariate partial least-squares (PLS) analysis (McIntosh et al., 2004) to examine the correlation between MFG volume and task- 
Table 1. Group means for education and neuropsychological tests

\begin{tabular}{|c|c|c|c|c|c|c|c|c|}
\hline Group & EDU (years) & LSBQ & MMSE & BECK & GDS & LFCVLT & LCRCVLT & $\overline{\text { RGCVLT }}$ \\
\hline \multicolumn{9}{|c|}{ Young adults } \\
\hline Mean & 16.08 & 2.08 & 28.32 & 2.60 & 0.96 & $13.57^{*}$ & 13.04 & 15.30 \\
\hline SE & 0.28 & 0.15 & 1.20 & 0.80 & 0.30 & 0.43 & 0.05 & 0.24 \\
\hline \multicolumn{9}{|c|}{ Older adults } \\
\hline Mean & 15.16 & 1.88 & 29.48 & 4.60 & 1.24 & $10.96^{*}$ & 11.61 & 15.00 \\
\hline SE & 0.48 & 0.18 & 0.16 & 0.88 & 0.32 & 0.67 & 0.68 & 0.28 \\
\hline
\end{tabular}

This table presents the group means and SEs for education and the other neuropsychological measures taken. LSBQ, Language and Social Background Questionnaire (Bialystok et al., 2005, 2007); EDU, education; LFCVLT, long-form CVLT; LCRCVLT, long form category assisted recall CVLT; RGCVLT, long-term recognition CVLT; MMSE, Mini-Mental Status Exam.

*Significant effect.

related whole-brain activity in both age groups. Then stepwise regression was used to determine which of these activated regions predicted retrieval accuracy in young and/or older adults.

\section{Materials and Methods}

We have previously published behavioral, neuropsychological, and univariate analyses of the fMRI data for a subset of the sample in the current study (21 young and 21 older adults) (Rajah et al., 2010). In the current study, we use a larger sample and present unique analyses and results pertaining to age-related changes in MFG volume, and how MFG volume relates to whole-brain patterns of retrieval activity and retrieval accuracy, using multivariate PLS and regression analyses.

\section{Subjects}

Twenty-five young (age range, 19-35 years; mean age, 23.72 years; mean education, 16.08 years; 13 males) and 26 older adults (age range, 60-80 years; mean age, 66.76 years; mean education, 15.16 years; 8 males) participated in the study. Volunteers were recruited from the community using local advertisements and referrals; all were right handed and fluent in English. We administered a battery of neuropsychological tests to exclude individuals suffering from depression and dementia (Rajah et al., 2010): the Mini-Mental Status Exam (cutoff score <27) (Folstein et al., 1975); the Beck Depression Inventory (Beck et al., 1961; Beck, 1987); Geriatric Depression Scale (Brink et al., 1982); the Cognitive Assessment Scale for the Older Adults (Geneau and Taillefer, 1996); and the California Verbal Learning Task (CVLT), using the long-form free recall, longform category-assisted free recall, and long-term recognition measures (Delis et al., 1987, 1988). One-way between-group ANOVAs were conducted on these variables and are presented in Table 1. No significant between-group differences were observed, except for long-form free recall performance on the CVLT, which is consistent with prior reports of age-related declines in free recall (Perlmutter, 1979).

In addition, a medical questionnaire was administered to ensure participants were psychologically, neurologically, and physically in good health. Moreover, anyone with a family member who had been diagnosed with Alzheimer's disease was excluded from the study. Additional medical exclusion criteria included diagnosis of diabetes, presence of cataracts or glaucoma, high cholesterol levels left untreated in the past 2 years, and high or low blood pressure left untreated in the past 2 years. All participants gave written informed consent. The study was approved by the ethics boards of the Douglas Hospital, the Montreal Neurological Institute (MNI), and McGill University.

\section{Behavioral methods}

Subjects were told that they would be participating in a computer-based visual nonverbal memory experiment in which the stimuli were blackand-white photographs of age-variant human faces, cropped from the neck upward, that had been rated as either neutral or pleasant by two independent raters. The stimuli have been used in several context memory tasks in the laboratory (Rajah et al., 2008, 2010), and details about the stimuli can be found in Rajah et al. $(2008,2010)$. The set of stimuli used for each memory task was balanced for age, gender, and neutral/pleasantness rating. E-Prime (Psychology Software Tools) was used to present the behavioral protocol and collect accuracy and reaction time (RT; in milliseconds). All motor responses were made with the subject's right (dominant) hand.

During the experiment, subjects were presented with lists of faces and were asked to intentionally encode these stimuli to perform subsequent item recognition, spatial context, or temporal context memory retrieval tasks. The item recognition task was included as an episodic retrieval "control" task for which performance was matched in young and older adults. The inclusion of this task allowed us to corroborate prior findings that age-related changes in episodic memory has a greater impact on older adults' performance on context versus item retrieval tasks, and allows us to determine whether context retrieval deficits may be more sensitive to function deficits in the right MFG compared with item recognition tasks. Subjects knew at encoding what type of retrieval task would follow. Each encoding list consisted of 12 unique faces, which were presented one at a time, for $2 \mathrm{~s}$ each, either to the left or right side of a fixation cross at the center of the computer monitor. As they were encoding, subjects were also asked to perform an orienting task in which they were asked to rate each face as pleasant (press button 1) or neutral (press button 2).

After each encoding list, there was a 1 min break during which subjects performed an alphabetizing distracter task. Each break was followed by (1) an item recognition task, (2) a spatial-context retrieval task, or (3) a temporal-context retrieval task. During item recognition, subjects were presented with one "new" face (not seen previously) and one "old" face (previously seen) and were asked to either select the old face or the new face, based on the retrieval cue presented above each stimulus pair. Each recognition event was $4 \mathrm{~s}$ in duration. During spatial context retrieval, subjects were presented with two old faces and asked to remember which face was presented either to the left or to the right of the central fixation cross, depending on the retrieval cue presented with each stimulus pair. During temporal context tasks, subjects were presented with two old faces and asked to remember which face was presented either "most" or "least" recently, depending on the retrieval cue presented with each stimulus pair. Each context retrieval event was $6 \mathrm{~s}$ in duration. The behavioral tasks were designed such that all retrieval tasks used a two-alternative forced choice structure, and two face stimuli were presented for all retrieval event types.

There was a total of 24 encoding blocks, all consisting of distinct face stimuli. Therefore, subjects performed eight recognition blocks, eight spatial context retrieval blocks, and eight temporal context retrieval blocks during the experiment. Each retrieval task consisted of 6 trials, yielding a total of 48 trials for each task type. The entire experiment lasted $\sim 90 \mathrm{~min}$. Detailed methods for this study are presented in Rajah et al. (2010).

\section{Behavioral analysis}

The RT data from this study have been described in detail previously (Rajah et al., 2010). Here we only report on the accuracy data. Grubb's test was used to identify outliers at $p<0.05$ (Barnett and Lewis, 1994). One older adult was identified as an outlier in terms of both recognition performance and right MFG volume outlier and was removed from all analyses described below. After excluding this outlier, SPSS was used to conduct group (2) by sex (2) by retrieval task type (3) repeated-measures ANOVA to examine accuracy (significance threshold $=p<0.05$ ). Sex was included as a covariate in the analysis as previous studies have reported better spatial memory performance in men (Herlitz et al., 1999; Nyberg et al., 2000c; Lewin et al., 2001). Post hoc between-group one-way ANOVAs were conducted to clarify the 2-by-3 ANOVA results. Post hoc polynomial contrasts were also conducted within group to determine whether there was a linear reduction in task accuracy from item recognition, to spatial context, to temporal context retrieval, which would be indicative of a linear change in task difficulty.

\section{MRI and fMRI methods}

Data acquisition. All MRI data were acquired on a 3 T Siemens Trio Scanner at the MNI. Subjects were asked to lie in a supine position, while structural and functional MRI scans were acquired with a standard head coil. A vacuum cushion was used to stabilize head motion. Structural scans were acquired using the Alzheimer's Disease Neuroimaging Initia- 
tive protocol parameters (Jack et al., 2008). This protocol generates T1weighted image volumes with a $1 \mathrm{~mm}$ isotropic resolution. The volumes were acquired using a fast 3D gradient echo sequence (acquisition time, 9 min $35 \mathrm{~s}$; TR, $14 \mathrm{~ms}$; TE, $4.92 \mathrm{~ms}$; flip angle, $25^{\circ}$; 160 1-mm-thick transverse slices; $1 \times 1 \times 1 \mathrm{~mm}$ voxels; FOV, $256 \mathrm{~mm}^{2}$ ).

Following the acquisition of the structural scan, participants performed the aforementioned behavioral tasks while blood oxygenation level-dependent (BOLD) (functional) images were acquired. A mixed, rapid, event-related experimental design was implemented for this experiment (Donaldson, 2004; Liu, 2004; Amaro and Barker, 2006). A variable intertrial interval (ITI) $(2-8 \mathrm{~s}$; mean ITI, $4.67 \mathrm{~s})$ served to add jitter to the fMRI acquisition sequence, allowing dissociation of eventrelated changes in BOLD activity. BOLD images were acquired using a single shot T2*-weighted gradient echo EPI pulse sequence (TR, $2000 \mathrm{~ms}$; TE, $30 \mathrm{~ms}$; FOV , $256 \mathrm{~mm}^{2}$; matrix size, $64 \times 64$; in-plane resolution, $4 \times$ $4 \mathrm{~mm} ; 340$ whole-brain acquisitions/11 min $20 \mathrm{~s}$ run). Each whole-brain acquisition consisted of 32 oblique slices of $4.0 \mathrm{~mm}$ thickness with no gap acquired along the anteroposterior commissural plane. Twenty seconds of gradient radiofrequency pulses preceded each experimental run to establish steady-state tissue magnetization and minimize startle-related movement during acquisition. Visual stimuli were generated by a PC laptop computer and back-projected via an LCD projector onto a screen placed at the foot of the scanner bed. The screen was made observable to participants via a mirror mounted within the head coil (visual angle,

$\sim 4^{\circ}$ ). Plastic optical corrective glasses were worn by participants who required correction for visual acuity. Participants used a fiber optic twobutton response box to perform the experimental tasks. The functional imaging portion of the study took $\sim 90 \mathrm{~min}$.

Data processing. Images were reconstructed from raw $k$-space, and between-slice time differences were corrected using a sinc interpolation method. Images were converted to ANALYZE format, and subsequent image processing was conducted using SPM2 software (http://www.fil. ion.ucl.ac.uk/spm/) run with MATLAB R2010b on a Linux platform. Images from the first $10 \mathrm{~s}$ of each run were discarded to control for field inhomogeneities. Functional images were spatially realigned to the first image acquired to correct for movement artifact using a six parameter rigid body spatial transform and a least-squares approach. Subjects with head motion $>3.5 \mathrm{~mm}$ were discarded from the analysis. Individual subject's functional images were spatially normalized to the MNI EPItemplate available in SPM2, placing them in a standard coordinate space based on the Talairach atlas (Talairach and Tournoux, 1988b), which facilitated group analysis and allowed for the reporting of activations in standard Talairach space (Collins et al., 1994; Ashburner and Friston, 2004). Volumes were resampled into $2 \mathrm{~mm}$ cubic voxels and smoothed using a $10 \mathrm{~mm}$ full-width at half-maximum isotropic Gaussian kernel to minimize interparticipant anatomic variability (Friston, 2004).

\section{MRI volumetric methods}

In vivo volumetric measurements of the left and right MFG were conducted by merging the results from the following two complimentary semiautomatic registration-based classification and segmentation methods developed at the MNI at McGill University: Automatic Nonlinear Image Matching and Anatomical Labeling (ANIMAL) and Intensity Normalized Stereotaxic Environment for the Classification of Tissue (INSECT) (Collins et al., 1999). First, all structural scans underwent signal-intensity normalization, noise reduction, and intensity nonuniformity correction, and were stereotaxically registered using nine parameters to the T1-weighted MNI template before volume segmentation. Thus, all subsequent volumetric measures are in standardized space and corrected for individual differences in head size (Collins et al., 1994; Collins and Evans, 1999; Zijdenbos et al., 2002). The automatic INSECT "pipeline" was used to classify each subject's MRI into GM, white matter (WM), and CSF (Zijdenbos et al., 2002). ANIMAL was used to automatically segment individual subject's MRI into all major cortical gyri (Collins and Evans, 1999). It maps major gyri and sulci across the whole brain of individual subjects by nonlinearly warping the individual's MRI to a template MRI; the International Consortium for Brain Mapping probabilistic atlas of the human brain is based on the MRIs of 150 subjects (Collins and Evans, 1999; Collins et al., 1999; Mazziotta et al.,

\section{A}



B

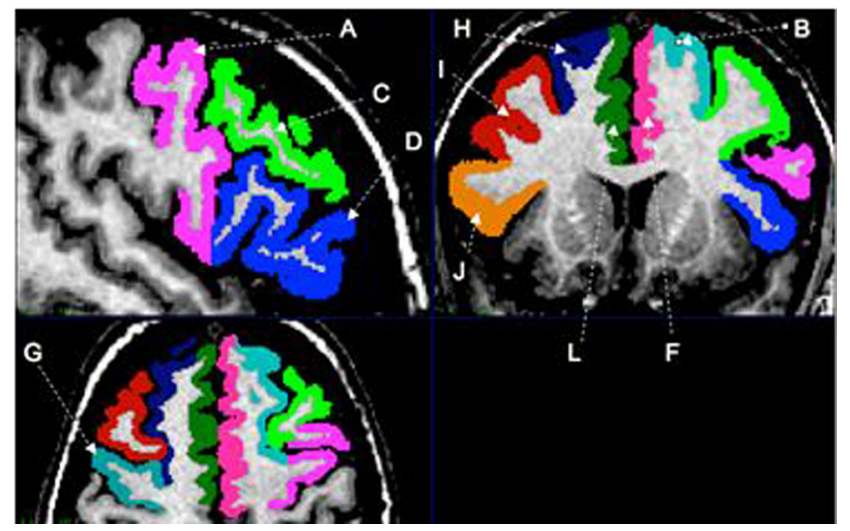

Figure 1. Anatomical markers for frontal regions of interest (ROIs). $A$, Three-dimensional rendered images with frontal regions of interest. Note that the cerebellum has been removed. 1, Frontal view: right $P \subset G(A)$, right superior frontal gyrus $(B)$, right middle frontal gyrus $(C)$, right inferior frontal gyrus $(D)$, right $0 F C(E)$, right medial cortex (F), left $P C G(G)$, left superior frontal gyrus $(H)$, left middle frontal gyrus (I), left inferior frontal gyrus (J), left OFC (K), left medial cortex (L). 2, Dorsal view. 3, Lateral view: same regional coding as in $\mathbf{1}$ applies. 4, Ventral view. Images taken from the Display program. $\boldsymbol{B}$, Two-dimensional working view showing a sagittal section of the right hemisphere (top left), a coronal section (top right), and transverse section (bottom left). The same letter coding from $\boldsymbol{A}$ applies for the respective frontal gray matter regions of interest. Image taken from the Display program.

2001).The anatomical segmentation of all major gyri and sulci results from ANIMAL were merged with the classification results from INSECT for each subject to accurately yield specific volumes for various cortical structures for each subject.

Automatic segmentation with ANIMAL has been shown to be successful for basal ganglia structures, but segmentation of the cortex has been less satisfactory. As such, we used a semiautomatic approach for segmenting the left and right MFG. Manual correction to the automated ANIMAL results for left and right MFG was conducted using the software package DISPLAY developed at the MNI (Collins et al., 1994). This program allows for simultaneous viewing in coronal, sagittal, and horizontal planes and a 3D surface rendering (Fig. 1). Demarcations that aided the manual correction of the segmented MFG were derived from Suzuki et al. (2005) and Tisserand et al. (2002). To isolate the MFG, the neighboring inferior frontal gyri (IFG), superior frontal gyri (SFG), precentral gyrus $(\mathrm{PcG})$, medial frontal cortex, and orbitofrontal cortex (OFC) were also 
segmented (see Fig. 1). Below we list the anatomical landmarks and guidelines used to segment the MFG.

\section{Middle frontal gyrus region of interest}

In defining the MFG, the ventrolateral border was considered to be the inferior frontal sulcus and the dorsomedial border was defined as the superior frontal sulcus. The posterior and ventral borders were defined as the precentral sulcus and the dorsal end of the OFC, respectively. The latter is the hardest rule to follow, because quite often the MFG will form a ventral boundary with the IFG directly without ever reaching the OFC (see Fig. 1 A, 1). The MFG encompasses mostly Brodmann areas (BAs) 46 and 9 , but can also consist of posterodorsal portions of BA10.

\section{MRI volumetric analyses}

Inter-rater and intrarater reliability assessment. After target structures were segmented in all subjects, volumes were extracted for statistical analysis from all individuals. Segmentation of all brains was done by a single rater (rater 1). A second rater (rater 2) also conducted segmentation of five brains to allow for inter-rater reliability assessments. Rater 1 also segmented five randomly selected brains a second time to allow for intrarater reliability assessment. Inter-rater and intrarater reliability assessments were conducted for left and right MFG using the Intraclass Correlation (ICC) $(3,1)$ standard of Shrout and Fleiss (1979) performed with SPSS (version 15). Both raters were blind to the age and identity of the participant. The time needed to completely segment one individual was $\sim 15 \mathrm{~h}$.

The inter-rater (and intrarater) ICC results for left MFG and right MFG were 0.99 (0.98) and 0.99 (0.99), respectively. The inter-rater (intrarater) results for left and right IFG and SFG were both also 0.99 (0.99). We report the reliability results for these additional regions of interest (ROIs) since we also conducted group-by-sex-by-hemisphere ANOVAs on these ROIs to assess the specificity of any results we obtained from the ANOVA conducted on MFG volumes. The high ICC values are not surprising given that the manual segmentations were mainly corrections to the results obtained from the automatic segmentation of the major gyri conducted using ANIMAL. Therefore, the PFC volumes reported here are highly reliable and replicable.

Group-by-sex-by-hemisphere volumetric analysis. To test the hypothesis that healthy aging was related to a disproportionate GM volume loss in right versus left MFG, we conducted a group (2) by sex (2) by hemisphere (2) repeated-measures ANOVA in which the left and right MFG volumes for each subject were the dependent measures. Sex was included as an independent variable due to prior work that has identified sex differences and age-by-sex interactions in regional cortical volumes (Raz et al., 2004). Although our a priori region of interest was the MFG, to determine whether age-related hemispheric asymmetry in volume reductions was specific to the MFG we also conducted group-by-sexby-hemisphere ANOVA for the IFG and SFG ROIs for which we had inter-rater and intrarater reliability assessments.

\section{fMRI analysis - correlating brain activity with MFG volumes}

Multivariate spatiotemporal ST-PLS analysis was used to directly examine group similarities and differences in the association between right MFG volume and retrieval-related brain activity during the performance of the following three episodic memory tests: item recognition, spatial context retrieval, and temporal context retrieval (McIntosh et al., 2004). ST-PLS is a powerful tool for identifying whole-brain, task-related and behavior-related changes in brain activity (Addis et al., 2004; McIntosh et al., 2004; Lenartowicz and McIntosh, 2005; Vallesi et al., 2009). In the following paragraphs, we present a brief overview of ST-PLS methods; for a more detailed description of ST-PLS, we refer you to the article by McIntosh et al. (2004).

In the current study, we used PLS to examine the association between right MFG volume and retrieval-related brain activity from correct item recognition, spatial context, and temporal context retrieval trials. This analysis was conducted to determine whether age-related reductions in right MFG volume influenced the structure-function association within the right MFG and between the right MFG and other brain regions associated with episodic memory retrieval of faces in healthy adults. These include the bilateral occipitotemporal cortex, bilateral inferior parietal cortex, and ventrolateral PFC (VLPFC) (Rajah et al., 1999, 2010; Grady, 2002; Persson et al., 2006). To do this, we used the "Behavior/Seed" ST-PLS module (ST-BPLS) to identify patterns of whole-brain activity that covaried with right MFG volume (McIntosh et al., 2003). ST-BPLS identifies a set of mutually orthogonal latent variables (LVs) that maximally relate the retrieval-related brain activity to right MFG volume. To conduct this analysis, each subject's $3 \mathrm{D}$ event-related fMRI data matrix was converted to a 2D data matrix by "flattening" the temporal dimension $(t)$, so that time series of each voxel $(m)$ was stacked side by side across the columns of the data matrix (column dimension $=m^{\star} t$ ). In the current analysis, the temporal dimension (time series) consisted of eight time lags ( 1 time lag $=1 \mathrm{TR}$ or $2 \mathrm{~s}$ ) after a correct item recognition, correct spatial context retrieval, or correct temporal context retrieval event. This allowed for the full sampling of the hemodynamic response (HR) to each event type without relying on assumptions about the shape of the HR function. Time lags were normalized by converting to the percentage change from the first time lag. The jittered ITI and rate of stimulus presentation allowed for adequate separation of event-specific changes in HR.

A between-group data matrix (containing data from both groups) was cross-correlated with the vectors of right MFG volume for young and older adults, resulting in a correlation for each trial type, across subjects, for each voxel. Singular value decomposition was then applied to this correlation matrix to generate the LVs, which consist of a singular value, a singular image, and a correlation profile for right MFG volume. The correlation profile shows how individual differences in right MFG volume correlate with the pattern of brain activity identified in the singular image in young and older adults. The singular image indicates which brain voxels exhibit the strongest correlation in activity with right MFG volume in young and older adults at each time lag after event onset. A singular image consists of negative and positive brain saliences, which are numerical weights assigned to each voxel at each time lag and represent a spatiotemporal pattern of whole-brain activity for the $16 \mathrm{~s}$ analysis window (eight $2 \mathrm{~s}$ TRs/time lags after event-onset). Brain regions with positive voxel saliences are positively related to the correlation profile for right MFG volume depicted for young and older adults for a given LV, and those with negative voxel saliences are negatively related to the correlation profile. The singular value indicates the strength of the correlation between retrieval-related activity in all brain voxels and right MFG volume. Additional results obtained from the ST-BPLS analysis are "brain scores" that represent the degree to which each subject expresses the pattern of brain activity identified by the singular image in relation to its paired correlation profile for right MFG volume at each time lag.

The statistical significance of each LV pair was determined by conducting 500 permutation tests on the singular values, which represented the proportion of the covariance matrix accounted for by each LV pair (McIntosh et al., 1998, 1999, 2004; McIntosh and Lobaugh, 2004). The probability that the permuted singular values exceed the observed singular values was calculated, and only LVs for which this probability was $p<$ 0.05 were deemed significant. To identify robust and stable voxels within a singular image, a bootstrap analysis of SEs was conducted (Efron and Tibshirani, 1986). The bootstrap method allowed us to identify voxels that consistently contributed to the experimental effect within each LV. Only local maxima with bootstrap ratios (BSRs) $\pm \geq 3.5(p<0.0005)$ and a spatial extent $>20$ voxels were considered significant. All local maxima coordinates were converted to Talairach space, and the Talairach and Tournoux (1988a) atlas was used to localize these maxima and identify the BA label for each cluster of activity.

Since episodic retrieval in young adults has been associated with activity in both left and right MFG, and we observed a general volume loss across the PFC with age, we conducted a second PLS analysis using the left MFG volumes as the variable to be correlated with brain activity. This analysis was conducted to determine whether left MFG volume also correlated with increased activity in brain regions associated with episodic memory retrieval of faces in healthy adults (listed above), and whether this effect was altered with healthy aging. By examining results from the right MFG and left MFG ST-PLS analyses, we would be able to determine whether volumes in both regions were similarly or differentially associated with retrieval-related activity in young and in older adults. 


\section{Linear regression analysis_predicting accuracy from brain} activity

An additional goal of this study was to determine whether retrieval accuracy in young and older adults could be predicted by individual differences in retrieval activity in brain areas where retrieval activity was also correlated with right and/or left MFG volume. The expectation was that activity in brain regions related to right MFG volume but not left MFG volume would predict performance on the memory tasks. SPSS (version 15) was used to conduct within-group multiple regression analyses, one per memory task. The dependent variables in the multiple regression analyses were accuracy on the recognition, spatial context, and temporal context tasks. The predictor variables included in the young-adult models were activations from LV1 of the right MFG volume ST-BPLS analysis (see Results). This LV-identified brain regions in which retrieval-related activity was correlated with right MFG volume in young adults only. Ten predictor variables for the regression were selected from LV1 (see Table 4). These ROIs were peak activations in left and/or right PFC, which was an a priori ROI for the current study, or were objectively chosen because they were one of the top 10 ROIs regarding spatial extent of activation or $\mathrm{BSR}$. The BSR is an indicator of the region's contribution to the pattern of the LV, such that an ROI with a highly positive or negative BSR makes a stable and robust contribution to the LV.

The mean percentage signal change for a $3 \mathrm{~mm}$ cubic area surrounding the peak coordinate of each ROI was extracted for each subject and each event type (correct recognition and spatial context and temporal context retrieval events) across all time lags. The plots of the impulse response functions for these ROIs indicated that the activity in these regions peaked $4-8 \mathrm{~s}$ after event onset for each event type. Therefore, we used the sum of the percentage signal change from 4 to $8 \mathrm{~s}$ after event onset as the predictor variables in the regression models. To assess concerns about the impact of multicollinearity among ROIs in the regression analyses, we conducted tests for multicollinearity and used a backward stepwise regression procedure to identify the best model for explaining task accuracy (Cody and Smith, 1991). Specifically, we assessed the presence of multicollinearity by ensuring that neither the tolerance value $(t<0.2$, indicative of multicollinearity) nor the variance inflation factor (VIF; VIF $>5$ indicates multicollinearity) (Mason and Perreault, 1991; O'Brien, 2007) indicated multicollinearity. In addition, we examined the correlations among ROI values within group to verify that none of the predictor variables exhibited a correlation $\geq 0.80$ with any of the other variables included in the model (Mason and Perreault, 1991). We used the $R$-change and $F$-statistic change $(p>0.05)$ for assessing goodnessof-fit and for determining which of the competing models from the backward elimination process best predicted accuracy. If there was no change in $F$-statistic probability $(p>0.05)$ after removing a predictor variable, this indicated that the removed variable did not add any predictive value to the model and it was acceptable to remove it from the model. We only report the $\beta$-values for the reduced model that best fits the accuracy data per memory task.

The same method was used for regression models in the older adults. The 10 predictor variables were chosen from LV2 of the right MFG volume ST-BPLS analysis using the criteria mentioned above (see Table 5). This LV identified brain regions in which retrieval-related activity was correlated with right MFG volume in older adults only. The same objective method for selecting predictor ROI variables was used in older adults as described above for the young-adults' models.

We also conducted backward stepwise regression analyses using the top 10 activations in LV1 of the left MFG volume ST-BPLS analysis (see Table 6). The rationale for conducting this analysis was to determine whether regions from the network identified from the left MFG ST-PLS analysis predicted performance in young and older adults (see Results). That is, we wanted to determine whether correlations between brain activity and behavior were specific to the regions correlated with the right MFG as predicted. LV1 from this ST-PLS analysis identified brain regions that were differentially active between age groups as a function of larger left MFG volume; retrieval activity in positive salience brain regions was positively correlated with larger MFG volumes in young adults (and negatively correlated in older adults), and those with negative salience were positively correlated with MFG volumes in older adults (and nega-
Table 2. Mean accuracy data with SE

\begin{tabular}{llll}
\hline Accuracy & Recognition & Spatial context & Temporal context \\
\hline Young adults $(N=25)$ & $0.94(0.01)$ & $0.90(0.02)$ & $0.81(0.02)$ \\
Older adults $(N=25)$ & $0.94(0.01)$ & $0.79(0.02)$ & $0.68(0.02)$ \\
\hline
\end{tabular}

Values shown are the proportion correct per task type (SE).

tively in young adults). Since this single LV characterized both groups, the same predictor variables (10 ROIs from Table 6) were used in the regression analysis for both young and older adults.

\section{Results}

\section{Behavioral results}

The mean accuracy scores for each group, per retrieval task, are presented in Table 2. The group-by-sex-by-task repeatedmeasures ANOVA showed there was a significant group main effect $\left(F_{(1,46)}=23.81 ; p<0.001\right)$, task main effect $\left(F_{(2,92)}=\right.$ $123.05 ; p<0.001)$, and a significant group-by-task interaction $\left(F_{(2,92)}=13.84 ; p<0.001\right)$. The significant interaction effect resulted from a lack of significant difference in accuracy between age groups for recognition events $(F<1.0)$, but significant betweengroup differences in spatial context retrieval $\left(F_{(1,48)}=20.91 ; p<\right.$ $0.001)$ and in temporal context retrieval $\left(F_{(1,48)}=23.81 ; p<0.001\right)$. There was no significant sex main effect $(p<0.05)$, and there were no interactions involving sex significant.

The post hoc polynomial contrasts indicated that there was a linear decrease in task accuracy from item recognition, to spatial context retrieval, to temporal context retrieval in both age groups (Young, $F_{(1,24)}=48.55 ; p<0.001$; Old, $F_{(1,24)}=309.24 ; p<$ $0.001)$. Therefore, both age groups performed best on item recognition followed by spatial context retrieval and performed worst on temporal context retrieval.

\section{PFC volumetric results}

Table 3 presents the mean volumes with SE in cubic millimeters for all PFC ROIs in young and older adults. Table 4 presents the group-by-sex-by-hemisphere repeated-measures ANOVA results for MFG, IFG, and SFG. The results from these three ANOVAs indicate there were group main effects for all ROIs due to older adults exhibiting significantly smaller volumes. However, only in MFG was there a significant hemisphere main effect $(p<0.05)$ and a significant group-by-hemisphere effect $(p<0.05)$. Post hoc within-age group repeated-measures ANOVAs examining hemispheric differences in MFG volume indicated there was no significant hemispheric difference in left versus right MFG volume in young adults $\left(F_{(1,24)}=2.40 ; p>0.05\right)$, but there was a significant hemispheric difference in left versus right MFG volume in older adults $\left(F_{(1,24)}=12.83 ; p=0.001\right.$ (Fig. 2 ) with lower values in right $\mathrm{MFG}$.

There was no significant main effect for sex for any of the ROIs $(p>0.05)$, but there was a significant sex-by-hemisphere interaction for the MFG. Post hoc within-sex group repeated-measures ANOVAs indicated there was a significant hemispheric difference in MFG volume in males $\left(F_{(1,21)}=13.99 ; p=0.001\right)$ but not in females $\left(F_{(1,27)}=2.78 ; p=0.10\right)$. Figure 2 presents the mean volumes (in cubic millimeters) for left and right MFG for young and older adult males and females, respectively. These results show that males in general have smaller right MFG volumes versus left MFG volumes. In addition, older adults, especially older men, exhibit smaller right MFG volumes versus left MFG volumes compared with young adults. Given that the older adult age group consisted of more females $(N=17)$ than males $(N=8)$ and the younger adult group had approximately the same num- 
Table 3. PFC ROI mean volumes by age, group, and sex

\begin{tabular}{|c|c|c|c|c|c|c|}
\hline Region of interest & Left IFG & Right IFG & Left MFG & Right MFG & Left SFG & Right SFG \\
\hline \multicolumn{7}{|l|}{ Young adults } \\
\hline \multicolumn{7}{|l|}{ Male } \\
\hline Mean & $22,065.21$ & $22,045.36$ & $24,072.43$ & $23,294.14$ & $24,903.93$ & $25,063.21$ \\
\hline$N$ & 14 & 14 & 14 & 14 & 14 & 14 \\
\hline SE & 401.74 & 472.97 & 877.30 & 815.02 & 590.40 & 538.12 \\
\hline \multicolumn{7}{|l|}{ Female } \\
\hline Mean & $21,915.00$ & $22,163.91$ & $24,233.09$ & $24,198.36$ & $24,109.18$ & $24,328.36$ \\
\hline$N$ & 11 & 11 & 11 & 11 & 11 & 11 \\
\hline SE & 460.66 & 523.27 & 660.99 & 768.01 & 394.79 & 680.10 \\
\hline \multicolumn{7}{|l|}{ Total } \\
\hline Mean & 21,999.12 & $22,097.52$ & $24,143.12$ & $23,692.00$ & $24,554.24$ & $24,739.88$ \\
\hline$N$ & 25 & 25 & 25 & 25 & 25 & 25 \\
\hline SE & 296.80 & 343.80 & 560.20 & 563.94 & 375.22 & 422.12 \\
\hline \multicolumn{7}{|l|}{ Older adults } \\
\hline \multicolumn{7}{|l|}{ Male } \\
\hline Mean & $19,733.13$ & $19,424.13$ & $20,888.00$ & $18,813.25$ & $19,312.50$ & $19,548.88$ \\
\hline$N$ & 8 & & & & & \\
\hline SE & 806.97 & 690.72 & 980.60 & 774.23 & 892.66 & 981.73 \\
\hline \multicolumn{7}{|l|}{ Female } \\
\hline Mean & $19,760.24$ & $20,326.88$ & $21,899.29$ & $21,101.71$ & $22,562.29$ & $21,578.53$ \\
\hline$N$ & 17 & 17 & 17 & 17 & 17 & 17 \\
\hline SE & 452.36 & 420.04 & 380.76 & 597.36 & 519.51 & 647.44 \\
\hline \multicolumn{7}{|l|}{ Total } \\
\hline Mean & $19,751.56$ & $20,038.00$ & $21,575.68$ & $20,369.40$ & $21,522.36$ & $20,929.04$ \\
\hline$N$ & 25 & 25 & 25 & 25 & 25 & 25 \\
\hline SE & 391.85 & 363.18 & 405.89 & 514.97 & 540.81 & 563.32 \\
\hline
\end{tabular}

Values are given as the mean gray matter volume $\left(\mathrm{mm}^{3}\right)$ (SE) in bilateral frontal cortex regions of interest (MFG, IFG, and SFG), grouped by age (young adults and older adults) and sex. Total, Group mean and SE collapsed across sex.

Table 4. Group-by-sex-by-hemisphere repeated-measures ANOVA results for PFC ROls

\begin{tabular}{lccc}
\hline & \multicolumn{3}{l}{ Region of interest } \\
\cline { 2 - 4 } & MFG & IFG & SFG \\
\hline Main effects & $20.92^{* *}$ & $21.87^{* *}$ & \\
Group & $20.92^{* *}$ & $21.87^{* *}$ & $39.59^{* *}$ \\
Sex & 2.33 & $<1.0$ & 2.34 \\
$\quad$ Hemisphere & $16.93^{* *}$ & $<1.0$ & $<1.0$ \\
Interaction effects & & & \\
Group $\times$ hemisphere & $5.28^{*}$ & $<1.0$ & 1.17 \\
Group $\times$ sex & $<1.0$ & $<1.0$ & $7.74^{*}$ \\
Sex $\times$ hemisphere & $5.09^{*}$ & 1.84 & 1.24 \\
Group $\times$ sex $\times$ hemisphere & $<1.0$ & $<1.0$ & 1.51 \\
\hline
\end{tabular}

$F$-values ( $\mathrm{df}=1,42$ ) for significant effects are presented for the three ANOVAs conducted to determine whether there were group-by-gender-by-hemisphere effects in MFG, IFG, and SFG volumes. ${ }^{*}$ Significant at $p=0.001$; ${ }^{*}$ significant at $p=0.05$.

ber of males and females (14 males, 11 females); it is clear that the significant age-by-hemisphere interaction effect was not biased by the hemispheric differences in volume between the sexes. In fact, given the male/female ratio in the two groups, the sex-byhemisphere effect would have biased against finding an age-byhemisphere effect. Therefore, these data indicate that the significant group-by-hemisphere effect was due to older adults exhibiting disproportionately smaller volumes in right relative to left MFG, and that this effect was specific to MFG since it was not observed in IFG or SFG.

\section{PLS results: right MFG volume and retrieval-related brain activity}

The permutation results indicated there were two significant LVs $(p<0.05)$. Figure 2 presents the singular image for the first LV with the corresponding bar graph depicting the correlation between brain scores (the degree to which each subject exhibited the pattern of activity observed in the singular image) and right MFG volume, with $95 \%$ confidence intervals, for the young and the older adult age groups, respectively (correlation profile). The correlation profile indicates that this LV identifies a pattern of brain activity robustly correlated to right MFG volume only in young adults.

Table 5 presents the local maxima for the positive and negative brain salience identified for the first LV. The negative correlation profile in young adults indicates that negative salience brain regions (Fig. $3 A$, blue) were more active in young adults as a function of larger right MFG volume during successful memory retrieval, regardless of retrieval task type. These regions included the bilateral dorsolateral PFC (within the MFG ROI), bilateral inferior parietal cortex, bilateral ventral occipitotemporal regions, and left precuneus. These negative salience regions constitute several areas that have been postulated to be a part of a core episodic memory retrieval network (Nyberg et al., 2000a). Positive salience brain regions (Fig. $3 A$, yellow/orange) were less active in young adults as a function of larger right MFG volume during successful memory retrieval and included right VLPFC, bilateral putamen, and several bilateral occipital regions.

Figure 3 presents the singular image and correlation profile for the second LV. The correlation profile indicates that this LV identified a pattern of brain activity that was positively correlated with right MFG volumes in older adults alone. Table 6 presents the local maxima for positive and negative salience brain regions identified in this LV. Positive salience regions were more active in older adults as a function of larger right MFG volumes during successful retrieval events for all retrieval task types and included the right inferior parietal cortex, right precuneus, right ventromedial PFC, right caudate, bilateral posterior cingulate cortex, left posterior parahippocampal cortex, and left thalamus. Negative salience regions were less active in older adults as a function of larger right MFG volumes during successful retrieval events. These regions included bilateral dorsomedial and dorsal PFC, bilateral anterior PFC, bilateral anterior parahippocampal cortex, and bilateral occipitotemporal regions.

\section{PLS results: left MFG volume and retrieval-related brain activity}

The permutation results indicated there was one significant LV $(p<0.05)$. Figure 4 presents the singular image and correlation profile for the second LV. The correlation profile indicates that this LV identified a pattern of brain activity that was differentially related to left MFG volumes in young versus older adults. In young adults, larger left MFG volume was related to greater activity in positive salience brain regions and less activity in negative salience regions. The opposite was true for older adults. Positive salience regions included the left posterior temporal cortex, bilateral precentral and postcentral cortex, and a left DLPFC area anterior and medial to the one reported in Table 5. Negative salience regions primarily included right-lateralized areas of occipitotemporal cortex and VLPFC. Table 7 presents the local maxima for positive and negative salience brain regions identified in this $\mathrm{LV}$.

\section{Linear regression results using regions correlated with right MFG}

Young-adult models

Ten ROIs were selected from the right MFG PLS LV1 results to be included in the backward stepwise regression analyses for predicting accuracy on the memory tasks (Table 5, asterisk): bilateral inferior occipital cortex, bilateral inferior parietal cortex, bilateral DLPFC, right medial PFC, two right VLPFC ROIs, and left precuneus. 
A Singular Image

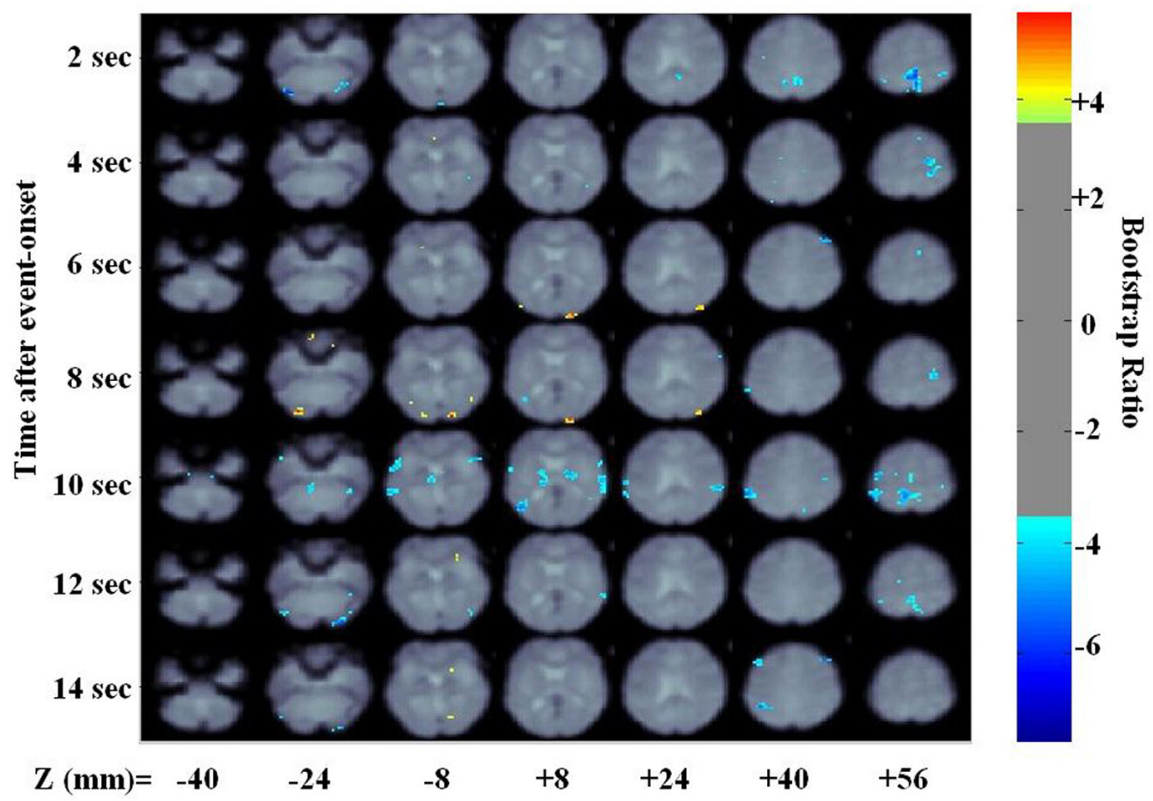

B Correlation Profile



Figure 2. Right MFG volume PLS result - first LV. $\boldsymbol{A}$, The singular image for the first $L V$ at a bootstrap ratio of $3.5(p<0.0005)$, which reflects stable and significant activations at $2-14 \mathrm{~s}$ after event onset. The $z$ coordinates are reported in millimeters in MNI space. The color bar represents the color code for the colorized activations on the singular image. Regions colored in red- orangeyellow are positively related with the experimental effect presented in $\boldsymbol{B}$. Regions colored in blue are negatively related to the experimental effect presented in $\boldsymbol{B}$. $\boldsymbol{B}$, Correlation profile for LV1. The correlation profile shows how right MFG volume correlated with the pattern of brain activity is identified in the singular image in young and older adults. RG, Recognition; S, spatial context; $T$, temporal context.

Item recognition. In young adults, the reduced model with only right inferior parietal cortex from LV1 was the best fit model for recognition accuracy $\left(F_{(1,23)}=7.69 ; p=0.01\right.$; adjusted $\left.R^{2}=0.25 ; \beta=+0.50\right)$. Since the right inferior parietal cortex exhibited greater activity in young adults with larger right MFG volume, the regression results indicate that greater activity in this area, in those with larger right MFG volumes, predicted better item recognition.

Spatial context retrieval. Spatial context regression analysis was conducted after excluding one young subject who performed with an accuracy $Z$-score $>1.96$ from the mean for this task alone. We reran the behavioral analyses listed above without this subject. Excluding this subject did not impact the overall behavioral results. A reduced model including left DLPFC $(\beta=+0.31)$ and left occipital cortex $(\beta=$ $+0.62)$ as positive predictors, and right VLPFC $(\beta=-0.48)$ and right occipital cortex $(\beta=-0.49)$ as negative predictors was the best model for spatial context accuracy $\left(F_{(4,19)}=4.53 ; p=0.01\right.$, adjusted $\left.R^{2}=0.38\right)$.

Young adults with larger right MFG volume exhibited greater activity in left DLPFC and less activity in right VLPFC (see Table 4). This increase in DLPFC and decrease in right VLPFC activity in individuals with larger right MFG volumes was beneficial to spatial context retrieval accuracy. On the other hand, young adults with larger right MFG volumes also had increased activity in the right ventral occipital cortex and less activity in the left ventral occipital cortex and right VLPFC (see Table 4). These patterns of activity, as a function of larger right MFG volumes, were detrimental to spatial context retrieval performance.

Temporal context retrieval. A reduced model including both right inferior parietal cortex $(\beta=+0.52)$ and left DLPFC $(\beta=+0.30)$ as significant positive predictors predicted temporal context accuracy in young adults $\left(F_{(2,22)}=5.66 ; p=0.01\right.$; adjusted $\left.R^{2}=0.34\right)$. Both right inferior parietal cortex and left DLPFC exhibited greater activity as a function of larger right MFG volume. The regression results indicate that greater activity in these regions also predicted better temporal context accuracy in young adults.

\section{Older-adult models}

Ten ROIs were selected from LV2 (see Table 5 for regions marked with asterisk) as predictors to be included in the backward stepwise regression analyses for retrieval accuracy in older adults. The regions included right inferior parietal cortex, right caudate, right dorsal MFG, bilateral anterior parahippocampal cortex, left superior temporal cortex, right middle temporal cortex, right anterior cingulate cortex and bilateral anterior PFC.

Item recognition. In older adults, a reduced model with only right anterior parahippocampal cortex as a negative predictor was the best model for predicting recognition accuracy $\left(F_{(1,23)}=\right.$ $7.80 ; p=0.01$, adjusted $R^{2}=0.22$; right $\left.\mathrm{BA}, 34 \beta=-0.50\right)$. Right parahippocampal cortex was less active in older adults with larger right MFG volume, and less activity in this region at retrieval was predictive of better recognition accuracy in older adults. 
Table 5. Local maxima for right MFG volume PLS—LV1

\begin{tabular}{|c|c|c|c|c|c|c|c|c|}
\hline \multirow[b]{2}{*}{ Temporal lag (s) } & \multirow[b]{2}{*}{ Bootstrap ratio } & \multirow[b]{2}{*}{ Spatial extent } & \multicolumn{3}{|c|}{ Talairach coordinates } & \multirow[b]{2}{*}{ HEM } & \multirow[b]{2}{*}{ Gyral location } & \multirow[b]{2}{*}{ BA } \\
\hline & & & $x$ & $y$ & $z$ & & & \\
\hline \multicolumn{9}{|c|}{ Positive salience brain regions } \\
\hline 4 & 3.69 & 38 & -4 & 27 & -8 & Left & Anterior cingulate & 32 \\
\hline 6 & 5.02 & 29 & 24 & -100 & 12 & Right & Middle occipital & 18 \\
\hline 6 & 4.18 & 29 & 36 & -88 & 27 & Right & Superior occipital & 19 \\
\hline 6 & 3.72 & 26 & 24 & 27 & -5 & Right & Inferior frontal & $47^{*}$ \\
\hline 8 & 5.29 & 20 & 20 & -93 & -2 & Right & Cuneus & 17 \\
\hline 8 & 5.00 & 23 & 20 & -100 & 12 & Right & Cuneus & 18 \\
\hline 8 & 4.66 & 33 & 32 & -84 & 26 & Right & Cuneus & 19 \\
\hline 12 & 4.53 & 47 & 16 & -70 & 3 & Right & Lingual & 18 \\
\hline 12 & 4.34 & 23 & 28 & 27 & -8 & Right & Inferior frontal & 47 \\
\hline 14 & 4.61 & 46 & 16 & -70 & 0 & Right & Lingual & 18 \\
\hline 2 & -4.52 & 59 & 44 & -44 & 54 & Right & Inferior parietal & 40 \\
\hline 2 & -4.08 & 60 & 48 & -23 & -2 & Right & Superior temporal & 22 \\
\hline 2 & -3.63 & 40 & 24 & -3 & 15 & Right & Putamen & \\
\hline 4 & -4.72 & 28 & -8 & -20 & 64 & Left & Medial frontal & 6 \\
\hline 4 & -3.73 & 22 & -55 & -65 & 14 & Left & Middle temporal & 19 \\
\hline 4 & -5.01 & 38 & 12 & 30 & 46 & Right & Superior frontal & 8 \\
\hline 4 & -4.84 & 172 & 20 & -9 & 48 & Right & Medial frontal & $6^{*}$ \\
\hline 4 & -4.07 & 20 & 16 & 2 & 33 & Right & Cingulate & 24 \\
\hline 4 & -3.88 & 30 & 16 & -29 & 38 & Right & Cingulate & 31 \\
\hline 4 & -3.73 & 22 & 55 & 4 & 3 & Right & Superior temporal & 22 \\
\hline 6 & -3.70 & 29 & -36 & -53 & 28 & Left & Middle temporal & 39 \\
\hline 6 & -5.65 & 32 & 44 & 37 & 35 & Right & Middle frontal & $9^{*}$ \\
\hline 6 & -3.89 & 24 & 12 & 10 & 51 & Right & Superior frontal & 6 \\
\hline 10 & -3.54 & 20 & 40 & 7 & -24 & Right & Superior temporal & 38 \\
\hline 12 & -5.37 & 158 & 0 & -47 & 61 & Left & Precuneus & 7 \\
\hline 12 & -4.40 & 22 & -16 & 15 & 58 & Left & Middle frontal & 6 \\
\hline 12 & -4.18 & 63 & -40 & -48 & 58 & Left & Inferior parietal & 40 \\
\hline 12 & -3.90 & 42 & -48 & -70 & 7 & Left & Middle occipital & 19 \\
\hline 12 & -3.52 & 24 & -16 & -13 & 52 & Left & Medial frontal & 6 \\
\hline 12 & -5.52 & 174 & 32 & -90 & -16 & Right & Inferior occipital & $18^{*}$ \\
\hline 12 & -4.26 & 57 & 67 & -39 & 6 & Right & Middle temporal & 22 \\
\hline 14 & -5.37 & 95 & -44 & -49 & 39 & Left & Inferior parietal & 40 \\
\hline 14 & -4.24 & 48 & -48 & 29 & 35 & Left & Middle frontal & $9^{*}$ \\
\hline 14 & -5.38 & 66 & 8 & 11 & 66 & Right & Superior frontal & 6 \\
\hline 14 & -5.23 & 34 & 44 & 33 & 35 & Right & Middle frontal & 9 \\
\hline 14 & -4.53 & 54 & 32 & -90 & -16 & Right & Inferior occipital & 18 \\
\hline
\end{tabular}

Temporal lag represents the time after event onset, when a cluster of voxels exhibited a peak bootstrap ratio reflecting the contrast effect of interest [episodic memory (EM) encoding vs retrieval main effect]. The bootstrap ratio threshold was set $t p= \pm \geq 3.5$, and identified dominant and stable activation clusters. The spatial extent refers to the total number of voxels included in the voxel cluster (threshold $=\geq 20$ ). The stereotaxic coordinates are measured in millimeters, and gyral location and BAs were determined by referring to Talairach and Tournoux (1988a,b). HEM, Cerebral hemisphere in which the activation occurred.

Spatial context retrieval. A reduced model with right middle temporal cortex as a positive predictor and right anterior cingulate cortex as a negative predictor was the best model for predicting spatial context retrieval accuracy $\left(F_{(2,22)}=4.01\right.$; $p=0.03$; adjusted $R^{2}=0.20$; right middle temporal $\beta=$ +0.57 ; right anterior cingulate $\beta=-0.55$ ). In both of these regions, activity decreased as a function of larger right MFG volume. Since activity in the right anterior cingulate was a negative predictor of performance, decreased activity in this region as a function of larger right MFG volume benefited performance on this task in older adults. In contrast, decreased activity in right middle temporal cortex as a function of larger right MFG volume was associated with reduced spatial context retrieval accuracy in older adults.

Temporal context retrieval. A significant regression model for predicting temporal context retrieval accuracy was not identified in older adults using the ROIs selected from LV2 (see Table 5). 


\section{A Singular Image}

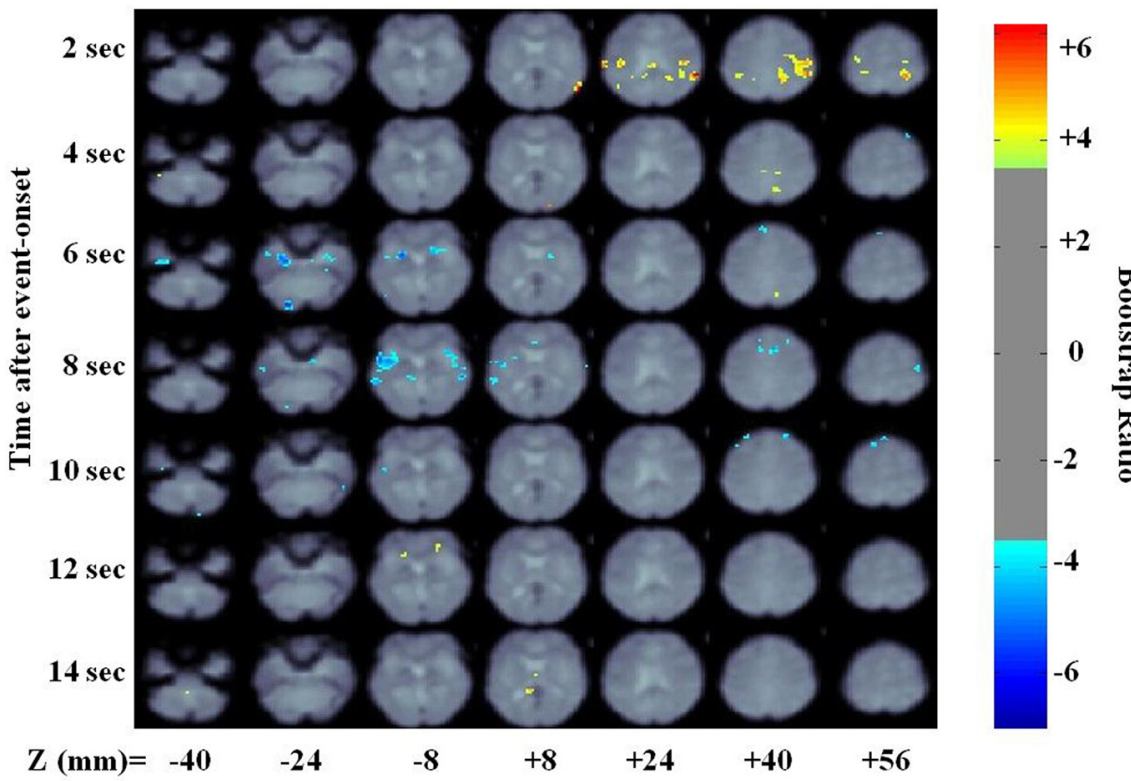

B Correlation Profile

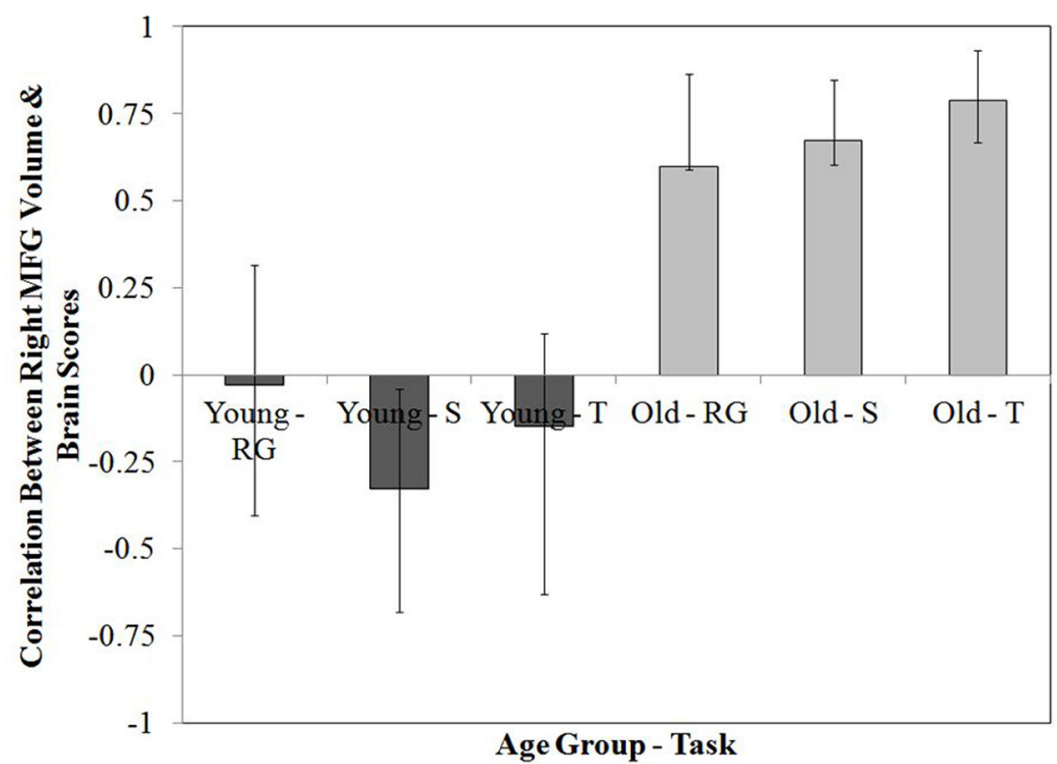

Figure 3. Right MFG volume PLS result-second LV. $A$, The singular image for LV2 at a bootstrap ratio of $3.5(p<0.0005)$, which reflects stable and significant activations at $2-14 \mathrm{~s}$ after event onset. The $z$ coordinates are reported in millimeters in MN space. The color bar represents the color code for the colorized activations on the singular image. Regions colored in red- orangeyellow are positively related with the experimental effect presented in $\boldsymbol{B}$. Regions colored in blue are negatively related to the experimental effect presented in $\boldsymbol{B}$. $\boldsymbol{B}$, Correlation profile for LV2. The correlation profile shows how right MFG volume correlated with the pattern of brain activity identified in the singular image in young and older adults. RG, Recognition; $\mathrm{S}$, spatial context; $T$, temporal context.

\section{Linear regression results using region correlated with left MFG}

Ten ROIs were selected from the left MFG PLS LV1 results to be included in the backward stepwise regression analyses for predicting accuracy on the memory tasks (Table 6, asterisk). These included the left anteromedial DLPFC, left anterior cingulate, bilateral PcG, left postcentral gyrus, left insula, right VLPFC, left cuneus, left middle temporal cortex, and right precuneus. The same ROIs were used to construct the young and older adult regression models.
Young-adult models

Item recognition. A reduced model with left precentral cortex and left insula as positive predictors was the best model for item recognition accuracy in young adults $\left(F_{(2,22)}=4.44 ; p=0.02\right.$; adjusted $R^{2}=$ 0.29 ; left precentral $\beta=+0.42$; left insula $\beta=+0.37$ ). In young adults, larger left MFG volume was related to increased activity in both of these ROIs during all retrieval tasks, and increased activity in these regions predicted better item recognition accuracy.

Spatial context retrieval. We again excluded a single young subject who performed with an accuracy $Z$-score $>1.96$ from the mean for this task alone (as mentioned above). Excluding this subject did not impact the overall behavioral results. A reduced model including the right precentral cortex $(\beta=-0.59)$, right precuneus $(\beta=+0.40)$, and left middle temporal gyrus $(\beta=+0.63)$ best predicted spatial context retrieval accuracy in young adults $\left(F_{(3,20)}=3.26\right.$; $p=0.04$; adjusted $R^{2}=0.23$ ). Thus, in young adults, having a larger left MFG volume was related to having greater activity in the right PcG and less activity in the right precuneus during retrieval, which negatively impacted spatial context retrieval accuracy. In contrast, having a larger left MFG volume was also related to greater left middle temporal cortex activity, which was related to increased spatial context retrieval accuracy.

Temporal context retrieval. A significant regression model for predicting temporal context retrieval accuracy was not identified in young adults using the ROIs selected from LV1 of the left MFG PLS analysis (see Table 6).

Older-adult models

Backward stepwise regression analyses using ROI activity from LV1 of the left MFG PLS analysis as predictors failed to yield a significant model for predicting performance accuracy on any of the retrieval tasks in older adults.

\section{Discussion}

The goal of this study was to determine whether healthy aging was associated with volumetric reductions in the right more than the left MFG GM and whether this volumetric deficit impacted patterns of retrieval-related brain activity and therefore context retrieval performance. Our behavioral results indicated that older adults performed significantly worse on context, but not item, retrieval tasks compared with young adults. This result is consistent with prior source/context memory studies of healthy aging (Craik et al., 1990; Glisky et al., 2001; Cabeza et al., 2002; Cansino et al., 2010). In the following sections, we discuss our volumetric find- 
Table 6. Local maxima for right MFG volume PLS—LV2

\begin{tabular}{|c|c|c|c|c|c|c|c|c|}
\hline \multirow[b]{2}{*}{ Temporal lag (s) } & \multirow[b]{2}{*}{ Bootstrap ratio } & \multirow[b]{2}{*}{ Spatial extent } & \multicolumn{3}{|c|}{ Talairach coordinates } & \multirow[b]{2}{*}{ HEM } & \multirow[b]{2}{*}{ Gyral location } & \multirow[b]{2}{*}{ BA } \\
\hline & & & $x$ & $y$ & $z$ & & & \\
\hline \multicolumn{9}{|c|}{ Positive salience brain regions } \\
\hline 2 & 6.24 & 2302 & 63 & -42 & 24 & Right & Inferior parietal & $40^{*}$ \\
\hline 4 & 3.80 & 20 & -8 & -29 & 38 & Left & Cingulate & 31 \\
\hline 4 & 4.87 & 56 & 12 & -64 & 36 & Right & Precuneus & 7 \\
\hline 4 & 3.88 & 25 & 16 & -33 & 35 & Right & Cingulate & 31 \\
\hline 6 & 4.59 & 30 & 12 & -64 & 36 & Right & Precuneus & 7 \\
\hline 12 & 4.95 & 285 & 20 & 27 & -1 & Right & Caudate* & \\
\hline 14 & 3.58 & 22 & 0 & -11 & 8 & Left & Thalamus & \\
\hline 14 & 4.37 & 133 & -8 & -38 & 9 & Left & Parahippocampal & 30 \\
\hline 14 & 4.67 & 31 & 16 & 46 & -16 & Right & Superior frontal & 11 \\
\hline \multicolumn{9}{|c|}{ Negative salience brain regions } \\
\hline 2 & -3.84 & 31 & -4 & 44 & 24 & Left & Medial frontal & 9 \\
\hline 2 & -4.21 & 61 & 48 & 25 & 43 & Right & Middle frontal & $8^{*}$ \\
\hline 4 & -4.03 & 38 & -48 & 7 & -14 & Left & Superior temporal & 38 \\
\hline 4 & -3.53 & 25 & -8 & 44 & 27 & Left & Medial frontal & 9 \\
\hline 4 & -4.22 & 26 & 36 & 34 & 50 & Right & Dorsal middle frontal & 8 \\
\hline 6 & -7.06 & 506 & -24 & -1 & -10 & Left & Parahippocampal & $34^{*}$ \\
\hline 6 & -5.57 & 65 & -24 & -86 & -16 & Left & Fusiform & 18 \\
\hline 6 & -4.45 & 35 & -48 & -70 & -7 & Left & Middle occipital & 19 \\
\hline 6 & -3.89 & 72 & -8 & 52 & 34 & Left & Medial frontal & 9 \\
\hline 6 & -4.97 & 423 & 28 & -1 & -13 & Right & Parahippocampal & $34^{*}$ \\
\hline 6 & -3.71 & 20 & 55 & -36 & -15 & Right & Inferior temporal & 20 \\
\hline 8 & -6.26 & 901 & -51 & -8 & 4 & Left & Superior temporal & $22^{*}$ \\
\hline 8 & -4.03 & 108 & -24 & -82 & -13 & Left & Fusiform & 19 \\
\hline 8 & -5.16 & 338 & 59 & -31 & -5 & Right & Middle temporal & $21^{*}$ \\
\hline 8 & -4.87 & 217 & 8 & 25 & 32 & Right & Cingulate & $32^{*}$ \\
\hline 8 & -4.65 & 27 & 24 & -78 & 1 & Right & Lingual & 18 \\
\hline 8 & -4.27 & 189 & 28 & -31 & 72 & Right & Postcentral & 3 \\
\hline 8 & -4.23 & 43 & 4 & 27 & 2 & Right & Anterior cingulate & 24 \\
\hline 10 & -5.14 & 278 & -12 & -8 & 67 & Left & Medial frontal & 6 \\
\hline 10 & -4.38 & 76 & 0 & 45 & 49 & Left & Superior frontal & 8 \\
\hline 10 & -4.36 & 64 & -32 & 52 & 31 & Left & Superior frontal & $9^{*}$ \\
\hline 10 & -4.31 & 169 & -48 & -12 & -3 & Left & Superior temporal & 22 \\
\hline 10 & -4.75 & 40 & 28 & 48 & 34 & Right & Superior frontal & $9^{*}$ \\
\hline 12 & -3.70 & 22 & -44 & 18 & -24 & Left & Superior temporal & 38 \\
\hline
\end{tabular}

Temporal lag represents the time after event onset, when a cluster of voxels exhibited a peak bootstrap ratio reflecting the contrast effect of interest (EM encoding vs retrieval main effect). The bootstrap ratio threshold was set $t p= \pm \geq 3.5$, and identified dominant and stable activation clusters. The spatial extent refers to the total number of voxels included in the voxel cluster (threshold $=\geq 20$ ). The stereotaxic coordinates are measured in millimeters, and gyral location and BAs were determined by referring to Talairach and Tournoux (1988a,b). HEM, Cerebral hemisphere in which the activation occurred.

ings, the association between MFG volume and retrieval activity and memory performance in young adults, and how healthy aging changes in these patterns of association.

\section{Age-related changes in right, more than left, MFG volume}

There was an overall GM volume reduction in all PFC gyri with aging. However, a significant age group-by-hemisphere interaction effect was only observed in the MFG. This was due to older adults exhibiting disproportionately smaller right versus left MFG compared with young adults.

Anatomically, it has been shown that the two cerebral hemispheres follow different developmental trajectories and that there are hemispheric differences in GM and WM density (Toga and Thompson, 2003; Sowell et al., 2004). In a volumetric study, Raz et al. (2004) directly examined age-related differences in hemispheric asymmetry in a variety of cortical regions, including the lateral PFC. They did not observe a significant hemispheric asymmetry in PFC volumes or a significant age-by-hemisphere interaction at a whole-brain level. However, in this earlier study the lateral PFC ROI included aspects of BAs $8,9,10,45$, and 46, and traversed across superior, middle, and inferior frontal gyri. In the current study, we examined volumes in the three main PFC gyri separately and observed more right volume loss than left with age only in the MFG. This volumetric result parallels prior fMRI reports of an age-related functional deficit in right MFG during memory retrieval (Rajah and D'Esposito, 2005; Rajah et al., 2010), which is consistent with a right MFG deficit hypothesis of healthy aging.

\section{Young adults: association among MFG volume, retrieval} activity, and performance

In young adults there was a positive association between larger right MFG volume and increased activity in a single "traditional" episodic memory network that included bilateral DLPFC, occipitotemporal, and inferior parietal cortices during all retrieval tasks (Rajah et al., 1999; Nyberg et al., 2000a). In contrast, larger left MFG volumes in young adults were positively correlated with greater activity primarily in temporal and motor regions and in the left anterior medial DLPFC. These results provide evidence that in young adults there is a direct positive association in MFG and surrounding DLPFC between structural and functional measures.

To determine whether activity in specific nodes of the episodic memory network and temporal and motor regions predicted retrieval accuracy in young adults, we conducted separate regression analyses in which the top 10 activated ROIs from the young right MFG PLS effect (see Table 5) and from the left MFG PLS effect (see Table 7) were included as predictors of performance on each retrieval task. Using ROIs from the right MFG PLS effect, we 


\section{A Singular Image}

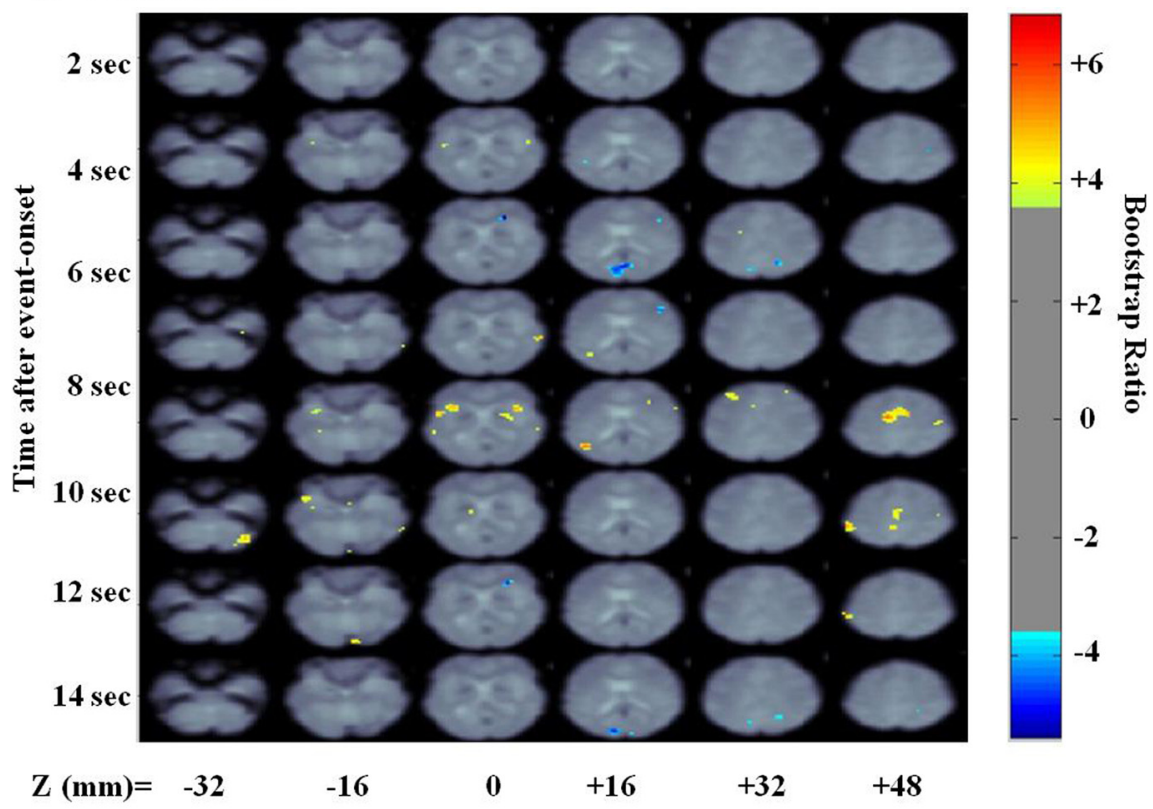

B Correlation Profile

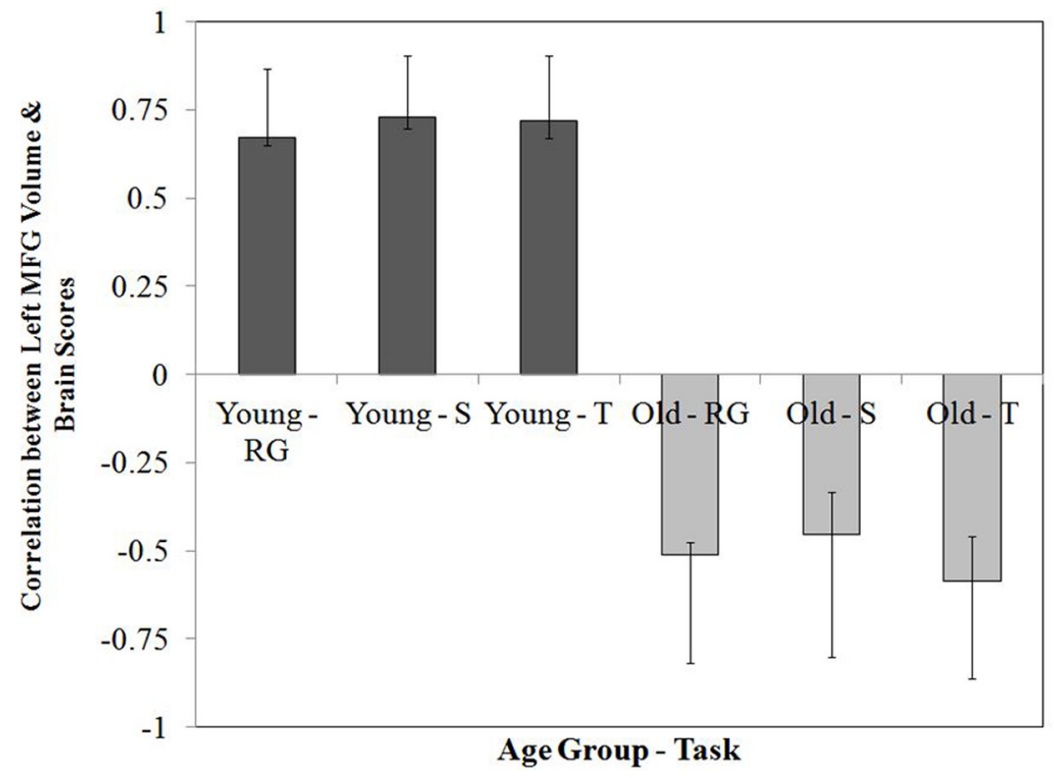

Figure 4. Left MFG volume PLS result-first LV. $A$, The singular image for LV1 at a bootstrap ratio of $3.5(p<0.0005)$, which reflects stable and significant activations at $2-14 \mathrm{~s}$ after event onset. The $z$ coordinates are reported in millimeters in $\mathrm{MNI}$ space. The color bar represents the color code for the colorized activations on the singular image. Regions colored in red-orange-yellow are positively related with the experimental effect presented in $\boldsymbol{B}$. Regions colored in blue are negatively related to the experimental effect presented in $\boldsymbol{B}$. $\boldsymbol{B}$, Correlation profile for LV1. The correlation profile shows how left MFG volume correlated with the pattern of brain activity identified in the singular image in young and older adults. RG, Recognition; $S$, spatial context; $T$, temporal context.

observed that right BA40 activity was a significant predictor of better item recognition and temporal context retrieval, and left BA9 activity was a positive predictor of both spatial and temporal context retrieval accuracy in young adults. Regression analyses involving ROIs from the left MFG PLS effect (see Table 7) indicated that item recognition accuracy was predicted by activity in the left PcG. Spatial context retrieval accuracy was predicted by activity in the right PcG. No significant model was observed for predicting temporal context accuracy using ROIs from the left MFG PLS effect.
Although we cannot determine from our current experimental design, prior studies of the specific cognitive processes mediated by distinct nodes of activity, such as left DLPFC, have emphasized the role of left DLPFC during successful context retrieval (Thaiss and Petrides, 2003; Dobbins et al., 2004; Mitchell et al., 2004, 2006), and have suggested that this region is involved in mediating cognitive control processes during memory retrieval, such as retrieval search, response selection, and/or postretrieval monitoring (Rajah et al., 2008). Similarly, several studies have reported greater inferior parietal cortex activity during retrieval success (Wagner et al., 2005). It has been proposed that the inferior parietal cortex is directly involved in retrieval via its role in bottom-up selective attention to mnemonic information retrieved by the hippocampus (Ciaramelli et al., 2008).

Together, our data indicate that in young adults there was a positive three-way association among right MFG volume, retrieval activity, and retrieval performance. Specifically, we found in young adults that the right but not the left MFG volume was positively correlated with increased activity in a single episodic memory retrieval network across all tasks (Nyberg et al., 2000), and that activity in specific nodes of this network (left DLPFC and right inferior parietal cortex) differentially predicted item and context retrieval in young adults only. Although the current study was not designed to compare and contrast single versus dual $/$ multiple process models of episodic retrieval, our results could be taken to support either model. For example, on the whole-brain network level, our results suggest that a single network of brain regions is engaged during both item and context retrieval in relation to right MFG volume, which supports a single process model. However, on the nodal level, we observed that increased activity in distinct nodes of this network differentially predicted item versus context retrieval, which supports a dual/multiple process model. Thus, our current results can support either model, depending on the level of analysis explored.

Age-related differences in the associations among right and left MFG volume, retrieval-related activity, and performance In our older adult sample, we did not observe a positive association between right MFG volume and increased retrieval activity in the episodic memory network, which was present in healthy young adults. Moreover, we did not observe a direct association between right MFG volume and activity in DLPFC. Instead, in older adults, larger right MFG volume was positively correlated with greater activity in the right parietal cortex, midline regions, 
Table 7. Local maxima for left MFG volume PLS—LV1

\begin{tabular}{|c|c|c|c|c|c|c|c|c|}
\hline \multirow[b]{2}{*}{ Temporal lag (s) } & \multirow[b]{2}{*}{ Bootstrap ratio } & \multirow[b]{2}{*}{ Spatial extent } & \multicolumn{3}{|c|}{ Talairach coordinates } & \multirow[b]{2}{*}{ HEM } & \multirow[b]{2}{*}{ Gyral location } & \multirow[b]{2}{*}{$B A$} \\
\hline & & & $x$ & $y$ & $z$ & & & \\
\hline \multicolumn{9}{|c|}{ Positive salience brain regions } \\
\hline 2 & 5.33 & 19 & -51 & -28 & 57 & Left & Postcentral & 2 \\
\hline 2 & 4.26 & 30 & -48 & -12 & -3 & Left & Superior temporal & 22 \\
\hline 6 & 4.40 & 15 & 28 & -32 & 64 & Right & Postcentral & 3 \\
\hline 6 & 4.13 & 29 & 24 & -9 & 56 & Right & Middle frontal & $6^{*}$ \\
\hline 8 & 6.13 & 34 & -44 & -65 & 14 & Left & Middle temporal & $39^{*}$ \\
\hline 8 & 5.58 & 405 & -16 & -5 & 48 & Left & Cingulate & $24^{*}$ \\
\hline 8 & 5.51 & 53 & -32 & 37 & 35 & Left & Middle frontal & $9^{*}$ \\
\hline 8 & 5.31 & 137 & -36 & 11 & -4 & Left & Insula & $13^{*}$ \\
\hline 8 & 4.13 & 19 & 24 & -4 & 0 & Right & Putamen & Putamen \\
\hline 10 & 6.87 & 540 & -24 & -12 & 63 & Left & Precentral & $6^{*}$ \\
\hline 10 & 5.88 & 101 & -40 & -40 & 61 & Left & Postcentral & $5^{*}$ \\
\hline 10 & 4.60 & 28 & 48 & -21 & 53 & Right & Postcentral & 3 \\
\hline 10 & 4.11 & 25 & -48 & 15 & -14 & Left & Superior temporal & 38 \\
\hline 12 & 4.89 & 35 & 4 & -47 & 61 & Right & Precuneus & 7 \\
\hline \multicolumn{9}{|c|}{ Negative salience brain regions } \\
\hline 2 & -4.30 & 18 & 36 & -21 & 53 & Right & Precentral & 4 \\
\hline 4 & -4.95 & 100 & 4 & -69 & 18 & Right & Precuneus & $31^{*}$ \\
\hline 6 & -5.18 & 19 & 24 & -96 & 16 & Right & Middle occipital & 19 \\
\hline 8 & -5.07 & 15 & 24 & -100 & 12 & Right & Middle occipital & 18 \\
\hline 12 & -5.38 & 21 & 28 & 27 & -5 & Right & Inferior frontal & $47^{*}$ \\
\hline 14 & -4.90 & 28 & -8 & -88 & 19 & Left & Cuneus & $18^{*}$ \\
\hline
\end{tabular}

Temporal lag represents the time after event-onset, when a cluster of voxels exhibited a peak bootstrap ratio reflecting the contrast effect of interest (EM encoding vs retrieval main effect). The bootstrap ratio threshold was set $t p= \pm \geq 3.5$ and identified dominant and stable activation clusters. The spatial extent refers to the total number of voxels included in the voxel cluster (threshold $=\geq 20$ ). The stereotaxic coordinates are measured in millimeters, and gyral location and BAs were determined by referring to Talairach and Tournoux (1988a,b). HEM, Cerebral hemisphere in which the activation occurred.

and right caudate. Previous studies have reported positive associations between posterior midline cortical activity and improved retrieval success (Daselaar et al., 2009) and between posterior midline cortical and caudate activity during declarative retrieval (Burianova et al., 2010). Therefore, these activations may reflect a change in the retrieval network associated with right MFG volume with age. However, in the current study we failed to observe any association between activity in these midline cortical and right caudate regions and retrieval accuracy in older adults.

Older adults also exhibited a negative association between right MFG volume and retrieval activity in several brain regions, including the right parahippocampal gyrus and anterior cingulate. The parahippocampal cortex has been hypothesized to play an important role in encoding and retrieving spatial information (Ekstrom and Bookheimer, 2007). In humans, the anterior cingulate has been implicated in conflict resolution during memory retrieval via the inhibition of extraneous mnemonic information (Fleck et al., 2006; Staudigl et al., 2010). Interestingly, we observed that decreased activity in the right parahippocampal gyrus during recognition and decreased activity in the right anterior cingulate during spatial context retrieval was beneficial to retrieval accuracy during these two tasks in older adults, respectively.

It has been suggested that one of the mechanisms underlying memory difficulties in older adults is that they encode too many extraneous associations, because they are distracted by irrelevant information (Campbell et al., 2010). During all encoding tasks stimuli were presented either to the right or left side of the computer screen; however, this spatial information was irrelevant for successful item recognition. Although speculative, one potential interpretation of the current findings is that older adults with larger right MFG were better able to alter activity in brain regions important for retrieving irrelevant spatial information (reflected in lower parahippocampal activity) and thus experienced less conflict in making memory judgments (reflected in lower anterior cingulate activity). This in turn aided their performance on item recognition and spatial context retrieval.
In general, our results suggest that older adults with larger right MFG volume may have engaged different mechanisms during item recognition and spatial context retrieval, compared with young adults, to compensate for overall volume reduction in the right MFG and disturbance in the right MFG structure-function association. In contrast, although older adults with larger left MFG volume exhibited greater activity in right VLPFC and occipital cortex, greater activity in these regions did not predict memory performance on any tasks in older adults. This supports our hypothesis that there is a specific influence of right MFG on retrieval activity and memory performance, and age-related changes in right MFG structure and function negatively impacts episodic retrieval abilities with age.

\section{Conclusions}

We conclude that in young adults larger right, but not left, MFG volume was positively correlated with increased activity in the episodic memory network; activity in specific nodes of this network was associated with better item and context retrieval. However, in healthy older adults there was more right than left MFG volume loss and disrupted structure-function association between right MFG volume and retrieval activity in the episodic memory network, which may underlie the context retrieval deficits observed in older adults. However, older adults with larger right MFG volumes attempted to compensate for this change by altering activity in different brain regions, which aided their performance on item recognition and spatial context retrieval, but not temporal context retrieval. These results support the hypothesis that a right MFG deficit with healthy aging negatively impacts episodic retrieval.

\section{Notes}

Supplemental material for this article about the anatomical landmarks and guidelines used to segment PFC regions surrounding the MFG is available at https://docs.google.com/document/d/1z_Qlhib60kv5Pac0vBviZ8tsDFAqiECoxMRtJoP0vQ/edit?authkey $=$ CNfajIOO\&hl=en_US\#. This material has not been peer reviewed. 


\section{References}

Addis DR, McIntosh AR, Moscovitch M, Crawley AP, McAndrews MP (2004) Characterizing spatial and temporal features of autobiographical memory retrieval networks: a partial least squares approach. Neuroimage 23:1460-1471.

Amaro E Jr, Barker GJ (2006) Study design in fMRI: basic principles. Brain Cogn 60: 220-232.

Ashburner J Friston K (2004) Spatial normalization using basis functions. In: Human brain function, Ed 2 (Frackowiak RS, Friston K, Frith C, Dolan R, Price CJ, Zeki S, Ashburner J, Penny W, eds), pp 655-672. London: Elsevier Academic.

Barnett V, Lewis T (1994) Outliers in statistical data, Ed 3. New York: Wiley.

Beck AT (1987) Beck depression inventory. San Antonio, TX: The Psychological Corporation.

Beck AT, Ward CH, Mendelson M, Mock J, Erbaugh J (1961) An inventory for measuring depression. Arch Gen Psychiatry 4:561-571.

Brink T, Yesavage J, Lum O, Heersema P, Adey M, Rose T (1982) Screening tests for geriatric depression. Clin Gerontol 1:37-44.

Buckner RL, Snyder AZ, Sanders AL, Raichle ME, Morris JC (2000) Functional brain imaging of young, nondemented, and demented older adults. J Cogn Neurosci 12 [Suppl 2]:24-34.

Burianova H, McIntosh AR, Grady CL (2010) A common functional brain network for autobiographical, episodic, and semantic memory retrieval. Neuroimage 49:865-874.

Cabeza R (2002) Hemispheric asymmetry reduction in older adults: the HAROLD model. Psychol Aging 17:85-100.

Cabeza R, Anderson ND, Houle S, Mangels JA, Nyberg L (2000) Age-related differences in neural activity during item and temporal-order memory retrieval: a positron emission tomography study. J Cogn Neurosci 12:197-206.

Cabeza R, Anderson ND, Locantore JK, McIntosh AR (2002) Aging gracefully: compensatory brain activity in high-performing older adults. Neuroimage 17:1394-1402.

Campbell KL, Hasher L, Thomas RC (2010) Hyper-binding: a unique age effect. Psychol Sci 21:399-405.

Cansino S, Trejo-Morales P, Hernández-Ramos E (2010) Age-related changes in neural activity during source memory encoding in young, middle-aged and elderly adults. Neuropsychologia 48:2537-2549.

Ciaramelli E, Grady CL, Moscovitch M (2008) Top-down and bottom-up attention to memory: a hypothesis (AtoM) on the role of the posterior parietal cortex in memory retrieval. Neuropsychologia 46:1828-1851.

Cody RP, Smith JK (1991) Applied statistics and the SAS programming language, Ed 3. Englewood Cliffs, NJ: Prentice Hall.

Collins DL, Neelin P, Peters TM, Evans AC (1994) Automatic 3D intersubject registration of MR volumetric data in standardized Talairach space. J Comput Assist Tomogr 18:192-205.

Collins DL, Zijdenbos AP, Baare WFC, Evans A (1999) ANIMAL+INSECT: improved cortical structure segmentation. In: Lecture notes in computer science, Vol 1613, Information processing in medical imaging, 16th International Conference IPMI'99, Visegrad, Hungary, June 28-July 2, 1999, Proceedings (Kuba A, Sámal M, Todd-Pokropek A, eds), pp $210-$ 223. Heidelberg, Germany: Springer-Verlag.

Collins L, Evans AC (1999) Animal: automatic nonlinear image matching and anatomical labeling. In: Brain warping (Toga AW, ed), pp 133-142. San Diego: Academic.

Craik FI, Morris LW, Morris RG, Loewen ER (1990) Relations between source amnesia and frontal lobe functioning in older adults. Psychol Aging 5:148-151.

Daselaar SM, Prince SE, Dennis NA, Hayes SM, Kim H, Cabeza R (2009) Posterior midline and ventral parietal activity is associated with retrieval success and encoding failure. Front Hum Neurosci 3:13.

Delis DC, Cullum CM, Butters N, Cairns P (1988) Wechsler memory scalerevised and California verbal learning test: convergence and divergence. Clin Neuropsychol 2:188-196.

Delis DC, Kramer JH, Kaplan E, Ober BA (1987) The California verbal learning test-research edition. San Antonio, TX: Psychological Corporation.

Dobbins IG, Rice HJ, Wagner AD, Schacter DL (2003) Memory orientation and success: separable neurocognitive components underlying episodic recognition. Neuropsychologia 41:318-333.

Dobbins IG, Simons JS, Schacter DL (2004) fMRI evidence for separable and lateralized prefrontal memory monitoring processes. J Cogn Neurosci 16:908-920.

Donaldson DI (2004) Parsing brain activity with fMRI and mixed designs: what kind of a state is neuroimaging in? Trends Neurosci 27:442-444.

Duarte A, Henson RN, Graham KS (2008) The effects of aging on the neural correlates of subjective and objective recollection. Cereb Cortex 18:2169-2180.

Efron B, Tibshirani R (1986) Bootstrap methods for standard errors, confidence intervals, and other measures of statistical accuracy. Stat Sci 1:54-75.

Ekstrom AD, Bookheimer SY (2007) Spatial and temporal episodic memory retrieval recruit dissociable functional networks in the human brain. Learn Mem 14:645-654.

Fleck MS, Daselaar SM, Dobbins IG, Cabeza R (2006) Role of prefrontal and anterior cingulate regions in decision-making processes shared by memory and nonmemory tasks. Cereb Cortex 16:1623-1630.

Folstein MF, Folstein SE, McHugh PR (1975) "Mini Mental State"—a practical method for grading the cognitive state of patients for the clinician. J Psychiatr Res 12:189-198.

Friston K. (2004) Experimental design and statistical parametric mapping. In: Human brain function, Ed 2 (Frackowiak RS, Friston K, Frith CD, Dolan RJ, Price CJ, Zeki S, Ashburner J, Penny W, ed), pp 599-634. London: Elsevier Academic.

Geneau D, Taillefer D (1996) Le "protocole d'examen cognitif de la personne âgée-version révisée" (PECPA-2r): normalisation par groupes d'âge et antécédents éducationnels chez des sujets québécois francophones. Paper presented at the Colloque de Psychogériatrie du C.C.F.P, St-Hyacinthe, Québec, Canada.

Glisky EL, Rubin SR, Davidson PS (2001) Source memory in older adults: an encoding or retrieval problem? J Exp Psychol Learn Mem Cogn 27:1131-1146.

Grady CL (2002) Age-related differences in face processing: a meta-analysis of three functional neuroimaging experiments. Can J Exp Psychol $56: 208-220$.

Hayama HR, Rugg MD (2009) Right dorsolateral prefrontal cortex is engaged during post-retrieval processing of both episodic and semantic information. Neuropsychologia 47:2409-2416.

Herlitz A, Airaksinen E, Nordström E (1999) Sex differences in episodic memory: the impact of verbal and visuospatial ability. Neuropsychology 13:590-597.

Jack CR Jr, Bernstein MA, Fox NC, Thompson P, Alexander G, Harvey D, Borowski B, Britson PJ, L Whitwell J, Ward C, Dale AM, Felmlee JP, Gunter JL, Hill DL, Killiany R, Schuff N, Fox-Bosetti S, Lin C, Studholme C, DeCarli CS, et al. (2008) The Alzheimer's Disease Neuroimaging Initiative (ADNI): MRI methods. J Magn Reson Imaging 27:685-691.

Jernigan TL, Archibald SL, Fennema-Notestine C, Gamst AC, Stout JC, Bonner J, Hesselink JR (2001) Effects of age on tissues and regions of the cerebrum and cerebellum. Neurobiol Aging 22:581-594.

Kaller CP, Rahm B, Spreer J, Weiller C, Unterrainer JM (2011) Dissociable contributions of left and right dorsolateral prefrontal cortex in planning. Cereb Cortex 21:307-317.

Knoch D, Gianotti LR, Pascual-Leone A, Treyer V, Regard M, Hohmann M, Brugger P (2006) Disruption of right prefrontal cortex by lowfrequency repetitive transcranial magnetic stimulation induces risktaking behavior. J Neurosci 26:6469-6472.

Lenartowicz A, McIntosh AR (2005) The role of anterior cingulate cortex in working memory is shaped by functional connectivity. J Cogn Neurosci 17:1026-1042.

Lewin C, Wolgers G, Herlitz A (2001) Sex differences favoring women in verbal but not in visuospatial episodic memory. Neuropsychology $15: 165-173$

Liu TT (2004) Efficiency, power, and entropy in event-related fMRI with multiple trial types. Part II: design of experiments. Neuroimage 21:401-413.

Mason CH, Perreault WD (1991) Collinearity, power, and interpretation of multiple regression analysis. J Mark Res 28:268-280.

Mazziotta J, Toga A, Evans A, Fox P, Lancaster J, Zilles K, Woods R, Paus T, Simpson G, Pike B, Holmes C, Collins L, Thompson P, MacDonald D, Iacoboni M, Schormann T, Amunts K, Palomero-Gallagher N, Geyer S, Parsons L, et al. (2001) A four-dimensional probabilistic atlas of the human brain. J Am Med Inform Assoc 8:401-430.

McIntosh AR, Lobaugh NJ (2004) Partial least squares analysis of 
neuroimaging data: applications and advances. Neuroimage 23 [Suppl 1]:S250-S263.

McIntosh AR, Lobaugh NJ, Cabeza R, Bookstein FL, Houle S (1998) Convergence of neural systems processing stimulus associations and coordinating motor responses. Cereb Cortex 8:648-659.

McIntosh AR, Rajah MN, Lobaugh NJ (1999) Interactions of prefrontal cortex related to awareness in sensory learning. Science 284: 1531-1533.

McIntosh AR, Rajah MN, Lobaugh NJ (2003) Functional connectivity of the medial temporal lobe relates to learning and awareness. J Neurosci 23:6520-6528.

McIntosh AR, Chau WK, Protzner AB (2004) Spatiotemporal analysis of event-related fMRI data using partial least squares. Neuroimage 23:764-775.

Mitchell KJ, Johnson MK, Raye CL, Greene EJ (2004) Prefrontal cortex activity associated with source monitoring in a working memory task. J Cogn Neurosci 16:921-934.

Mitchell KJ, Raye CL, Johnson MK, Greene EJ (2006) An fMRI investigation of short-term source memory in young and older adults. Neuroimage 30:627-633.

Nyberg L, Persson J, Habib R, Tulving E, McIntosh AR, Cabeza R, Houle S (2000a) Large scale neurocognitive networks underlying episodic memory. J Cogn Neurosci 12:163-173.

Nyberg L, Habib R, McIntosh AR, Tulving E (2000b) Reactivation of encoding-related brain activity during memory retrieval. Proc Natl Acad Sci U S A, 97:11120-11124.

Nyberg L, Habib R, Herlitz A (2000c) Brain activation during episodic memory retrieval: sex differences. Acta Psychol (Amst) 105:181-194.

O'Brien RM (2007) A caution regarding rules of thumb for variance inflation factors. Qual Quant 41:673-690.

Perlmutter M (1979) Age differences in adults' free recall, cued recall, and recognition. J Gerontol 34:533-539.

Persson J, Nyberg L, Lind J, Larsson A, Nilsson LG, Ingvar M, Buckner RL (2006) Structure-function correlates of cognitive decline in aging. Cereb Cortex 16:907-915.

Rajah MN, McIntosh AR, Grady CL (1999) Frontotemporal interactions in face encoding and recognition. Brain Res Cogn Brain Res 8:259-269.

Rajah MN, Ames B, D’Esposito M (2008) Prefrontal contributions to domain-general executive control processes during temporal context retrieval. Neuropsychologia 46:1088-1103.

Rajah MN, Languay R, Valiquette L (2010) Age-related changes in prefrontal cortex activity are associated with behavioural deficits in both temporal and spatial context memory retrieval in older adults. Cortex 46:535-549.

Rajah MN, D'Esposito M (2005) Region-specific changes in prefrontal function with age: a review of PET and fMRI studies on working and episodic memory. Brain 128:1964-1983.

Raz N, Gunning-Dixon F, Head D, Rodrigue KM, Williamson A, Acker JD
(2004) Aging, sexual dimorphism, and hemispheric asymmetry of the cerebral cortex: replicability of regional differences in volume. Neurobiol Aging 25:377-396.

Raz N, Lindenberger U, Rodrigue KM, Kennedy KM, Head D, Williamson A, Dahle C, Gerstorf D, Acker JD. (2005) Regional brain changes in aging healthy adults: general trends, individual differences and modifiers. Cereb Cortex 15:1676-1689.

Shrout PE, Fleiss JL (1979) Intraclass correlations: uses in assessing rater reliability. Psychol Bull 86:420-428.

Slotnick SD, Moo LR (2006) Prefrontal cortex hemispheric specialization for categorical and coordinate visual spatial memory. Neuropsychologia 44:1560-1568.

Sowell ER, Thompson PM, Toga AW (2004) Mapping changes in the human cortex throughout the span of life. Neuroscientist 10:372-392.

Staudigl T, Hanslmayr S, Bäuml KH (2010) Theta oscillations reflect the dynamics of interference in episodic memory retrieval. J Neurosci 30:11356-11362.

Suzuki M, Zhou SY, Takahashi T, Hagino H, Kawasaki Y, Niu L, Matsui M, Seto H, Kurachi M (2005) Differential contributions of prefrontal and temporolimbic pathology to mechanisms of psychosis. Brain 128:2109-2122.

Talairach J, Tournoux P (1988a) Co-planar stereotaxic atlas of the human brain (Rayport M, translator). New York: Thieme Medical Publishers.

Talairach J, Tournoux P. (1988b) Co-planar stereotaxic atlas of the human brain: 3-dimensional proportional system-an approach to cerebral imaging. New York: Thieme Medical Publishers.

Thaiss L, Petrides M (2003) Source versus content memory in patients with a unilateral frontal cortex or a temporal lobe excision. Brain 126:1112-1126.

Tisserand DJ, Pruessner JC, Sanz Arigita EJ, van Boxtel MP, Evans AC, Jolles J, Uylings HB. (2002) Regional frontal cortical volumes decrease differentially in aging: an MRI study to compare volumetric approaches and voxel-based morphometry. Neuroimage 17:657-669.

Toga AW, Thompson PM (2003) Mapping brain asymmetry. Nat Rev Neurosci 4:37-48.

Tulving E (1984) Précis of elements of episodic memory. Behav Brain Sci 7:223-268.

Vallesi A, McIntosh AR, Alexander MP, Stuss DT (2009) FMRI evidence of a functional network setting the criteria for withholding a response. Neuroimage 45:537-548.

Wagner AD, Shannon BJ, Kahn I, Buckner RL (2005) Parietal lobe contributions to episodic memory retrieval. Trends Cogn Sci 9:445-453.

West R (2000) In defense of the frontal lobe hypothesis of cognitive aging. J Int Neuropsychol Soc 6:727-729.

Zijdenbos AP, Forghani R, Evans AC (2002) Automatic "pipeline" analysis of 3-D MRI data for clinical trials: application to multiple sclerosis. IEEE Trans Med Imaging 21:1280-1291. 